\title{
Spatial noise-field control with online secondary path modelling: A wave-domain approach
}

\author{
Wen Zhang, Member, IEEE, Christian Hofmann, Michael Buerger, \\ Thushara D. Abhayapala, Senior Member, IEEE, and Walter Kellermann, Fellow, IEEE,
}

\begin{abstract}
Due to strong inter-channel interference in multichannel ANC, there are fundamental problems associated with the filter adaptation and online secondary path modelling remains a major challenge. This paper proposes a wave-domain adaptation algorithm for multichannel ANC with online secondary path modelling to cancel tonal noise over an extended region of $2 \mathrm{D}$ plane in a reverberant room. The design is based on exploiting the diagonal-dominance property of the secondary path in the wave domain. The proposed wave-domain secondary path model is applicable to both concentric and non-concentric circular loudspeaker and microphone array placement and is also robust against array positioning errors. Normalized least mean squarestype algorithms are adopted for adaptive feedback control. Computational complexity is analyzed and compared with the conventional time-domain and frequency-domain multichannel ANC. Through simulation-based verification in comparison with existing methods, the proposed algorithm demonstrates more efficient adaptation with low-level auxiliary noise.
\end{abstract}

Index Terms-Active noise control, secondary path modelling, wave domain, adaptive processing, feedback system

\section{INTRODUCTION}

Active noise control (ANC) is currently the most effective method for cancelling low-frequency noise. It is based on the principle of superposition, that is, an anti-noise signal of equal amplitude and opposite phase is generated by the secondary source to cause destructive interference and to cancel the primary noise [1], [2]. Single-channel ANC, known as one-dimensional ANC, is now a mature technique with several commercially successful applications, such as noisecancelling headphones, active mufflers, and the control of noise inside air conditioning ducts. Multichannel ANC on the other hand mainly deals with a noise field over space, thus also known as three-dimensional ANC, which requires several secondary sources and sensors, thus making it more complex and expensive to implement. An ANC system for

Wen Zhang is with Center of Intelligent Acoustics and Immersive Communications, School of Marine Science and Technology, Northwestern Polytechnical University, Xi'an, China (e-mail: wen.zhang@nwpu.edu.cn).

Thushara D. Abhayapala and Wen Zhang are with Research School of Engineering, College of Engineering and Computer Science, The Australian National University, Canberra, Australia (e-mail: thushara.abhayapala@anu.edu.au; wen.zhang@anu.edu.au).

Christian Hofmann, Michael Buerger, and Walter Kellermann are with Chair of Multimedia Communications and Signal Processing, Friedrich-Alexander University Erlangen-Nürnberg, Erlangen, Germany (e-mail: Christian.Hofmann@FAU.de; Michael.Buerger@FAU.de; Walter.Kellermann@FAU.de).

This work was supported by the National Natural Science Foundation of China (NSFC) funding scheme under Project No. 61671380 and the Australian Research Council (ARC) Discovery Projects funding scheme under Project DP140103412. the car interior [3] is a typical multichannel ANC application, which is capable of reducing short-time stationary noise, such as engine noise, at frequencies up to a few hundred Hertz [4], [5].

The general multichannel ANC system using the filtered$\mathrm{X}$ Least Mean Squares (LMS) algorithm was proposed in both time domain and frequency domain [6], [7], [8]. Its convergence behaviour was analyzed in the frequency domain in terms of the convergence of individual secondary signals, cost function, and control effort [9]. The computational complexity of multichannel ANC systems increases significantly with the number of secondary sources and error sensors, which represents one of the major challenges for applying multichannel ANC in large-scale applications [10]. In addition, in multichannel ANC, the noise control is achieved mainly at the error sensor positions or its close surroundings and noise still exists and may even be amplified at other positions [11]. However, in many practical ANC applications, especially in consumer electronics and medical instruments (e.g., magnetic resonance imaging (MRI) systems and infant incubators), it is desirable to create quiet zones away from error sensors.

A technique known as virtual sensing [12] was proposed. This technique requires a training stage to measure the system model from physical sensors to virtual sensors, which later on will be used in the online operation of the virtual sensing ANC system [13]. Obviously, the technique is highly dependent on the accuracy of the system model obtained during the training stage; any change of the acoustic environment or the physical sensor locations would result in system failure. Statistical knowledge of the noise field has been used for virtual sensing ANC without the offline training [14]; however, this is only effective for cancelling tonal diffusive noise fields. Still, noise cancellation essentially happens at physical sensor or virtual sensor positions. Therefore, the locations of reference and error sensors are very important to obtain the best estimate of the reference noise and total residual noise so as to achieve global noise cancellation or to create a large-sized quiet zone [15], [16].

Wave-domain signal processing, a technique commonly used for sound control over large spatial regions, provides a more efficient method for ANC over space [11], [17], [18], [19], [20], [21]. The principle of wave-domain signal representation is to use fundamental solutions of the Helmholtz wave-equation, or the eigen-solutions of the acoustic wave equation, as basis functions to express a wave field over a spatial region. Processing directly on the decomposition coefficients therefore controls sound within that region. The 
wave-domain ANC with feed-forward [18] and feedback [11], [22] configurations have been investigated and the results show that significant noise cancellation over the entire region of interest can be achieved.

In practical applications, the characteristics of the noise source, such as its frequency content, amplitude, and phase, are changing. The acoustic channel between the secondary source and error sensor, normally defined as the secondary path, must also be assumed to have a time-varying acoustic response as a result of a temperature variation or secondary source and error sensor position variations [23], [24]. To deal with such time-variance, adaptive filters such as the LMS or its variants, e.g., filtered-X LMS and recursive LMS [1], [2], [6-9], [25], are employed.

The performance of an ANC system thus strongly depends on the accurate estimation of the secondary path model [26]. The online secondary path modelling (SPM) technique using additive random noise was proposed [27], where a significant problem is the mutual interference between the noise control process and the secondary path modelling process. Methods to solve this problem include adding another adaptive filter into the system to remove the interference [28], [29], [30], improving the adaptive algorithm with the weight-average approach for the noise control filter [31], and controlling the power of additive noise and the step size in the online modelling process [32], [33]. However, in multichannel ANC, there are fundamental problems associated with the adaptation of the filters, such as high computational complexity and poor adaptation performance [34]. Especially, due to strongly cross-correlated inputs (or the well-known non-uniqueness and misalignment problem in multichannel acoustic systems, such as in acoustic echo cancellation [35]), the problem of online SPM in large-scale multichannel setup has hardly been addressed and most known works assume that the secondary path is known as a prior.

In this work, we propose an adaptive wave-domain ANC system with an effective strategy for online SPM based on the concepts outlined in [18], [36], [37], [38] for room equalization, acoustic echo cancellation, and ANC. The problem is defined as to cancel tonal noise (i.e., a single frequency or multi-frequency noise) on a $2 \mathrm{D}$ plane in a reverberant room, within which the secondary path is unknown ${ }^{1}$. The feedback control strategy is adopted with the loudspeakers and microphones used as the secondary sources and error sensors, respectively. Based on the wave-domain transform of the loudspeaker signals and microphone signals given in [39], we show that the wave-domain secondary path has the property of diagonal dominance for calibrated array placement on a quantitative level ${ }^{2}$. We then apply a systematic approach of SPM under a variety of positioning conditions,

\footnotetext{
${ }^{1}$ In practice, tonal noise is mainly generated by rotating equipment, such as engines and pumps. Active control of tonal noise within a small reverberant room, such as within the car interior, has received considerable attention. The practical relevance of this work is that it presents a spatial ANC system, which is capable of controlling tonal noise over space in a time-varying acoustic environment.

${ }^{2}$ Earlier related work has been published by the authors [40], in which only the situation of concentric loudspeaker and microphone array placement was considered.
}

i.e., concentric/non-concentric placement of the loudspeaker array and microphone array, and placement of loudspeaker$\mathrm{s} /$ microphones with random positioning errors. The adaptive algorithm using Normalized LMS (NLMS) is adopted for online SPM and ANC. It is shown that the proposed system requires only low-level auxiliary noise for online SPM and also demonstrates an efficient adaptation.

The state-of-the-art wave-domain ANC system is revisited in Sec. II. Section III demonstrates the diagonal-dominance property of the wave-domain secondary path and a systematic approach to model it under different positioning conditions. A feedback control strategy of the wave-domain ANC with online SPM is proposed in Sec. IV, and its computational complexity is analyzed. In Sec. V, the proposed system is evaluated in comparison with the existing frequency-domain and time-domain multichannel ANC systems.

\section{WAVE-DOMAIN ANC}

In this paper, we assume that the ANC system shown in Fig. 1 operates on a 2D plane within 3D physical space, e.g., at the height of a human ear. For ease of analytical treatment we also assume that the secondary sources (i.e. loudspeakers) and error sensors (i.e. microphones) are placed on two circular, not necessarily concentric, arrays. Other array topologies for wave-domain adaptive filtering have already been discussed in, e.g., [41], [42]. The aim here is to achieve noise cancellation within a $2 \mathrm{D}$ control region that is surrounded by the microphones while both the primary noise source and secondary source produce 3D variant sound fields (also known as 2.5D control in soundfield reproduction [43], [44] ${ }^{3}$.

Assuming $L$ secondary source signals $x_{\ell}(n), \ell=1, \ldots, L$, and $P$ error sensors, the received signals $y_{p}(n)$ at the microphones are written as

$$
y_{p}(n)=\sum_{\ell=1}^{L} x_{\ell}(n) * h_{p \ell}(n)+d_{p}(n), \quad p=1, \ldots, P,
$$

where $h_{p \ell}(n)$ is the impulse response of the secondary path from the $\ell$ th speaker to the $p$ th microphone, $*$ represents the time-domain convolution with respect to the time index $n$, and $d_{p}(n)$ is the recording of the primary noise at the $p$ th microphone.

It is assumed that the secondary path is time-varying and unknown; hence, the secondary source signal does not only consist of the anti-noise signal $x_{\ell}^{\mathrm{c}}(n)$, but it also contains an auxiliary noise signal $x_{\ell}^{\mathrm{e}}(n)$ for online SPM, i.e.,

$$
x_{\ell}(n)=x_{\ell}^{\mathrm{c}}(n)+x_{\ell}^{\mathrm{e}}(n),
$$

where $(\cdot)^{\mathrm{c}}$ and $(\cdot)^{\mathrm{e}}$ stand for cancellation and estimation, respectively. For the benefit of the listener, it is desirable to keep the level of the auxiliary noise minimum during online SPM.

\footnotetext{
${ }^{3}$ Note that an extension to a 3D control region is also possible. However, this would result in a significant increase in computational complexity due to the large number of transducers required for spherical arrays. Furthermore, the $2.5 \mathrm{D}$ formulation with a control region at the height of a human ear is a commonly used and practical setup in both soundfield reproduction [43], [44], [45], [46] and spatial ANC [11], [17], [21] applications.
} 


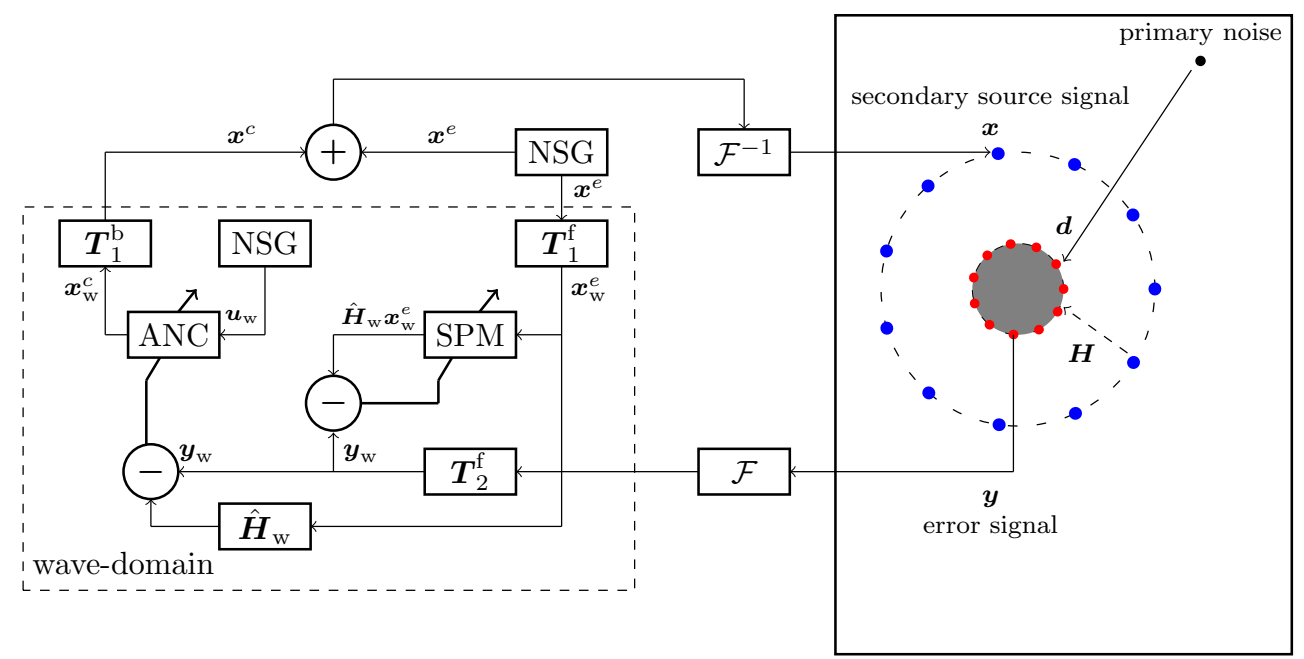

Fig. 1. System setup for a wave-domain ANC in a reverberant room. Uniform circular arrays of loudspeakers (blue) and microphones (red) are placed on a 2D plane for noise cancellation within the shaded region. Adaptive filters are adopted to deal with non-stationary noise and time-varying environment. Two independent noise sequences, one in the time domain and the other in the wave domain, are produced by the noise source generators (NSG) to drive the corresponding filter. The details are explained in Sec. IV-A and illustrated in Fig. 5.

We adopt a block-wise operation and transform the signal into the short-time Fourier transform (STFT) domain to obtain,

$$
Y_{p}(q, \omega)=\sum_{\ell=1}^{L} X_{\ell}(q, \omega) H_{p \ell}(q, \omega)+D_{p}(q, \omega),
$$

where $q$ and $\omega$ denote the $q$ th time block and the angular frequency, respectively.

Representing (3) in matrix form gives us

$$
\boldsymbol{y}(q, \omega)=\boldsymbol{H}(q, \omega) \boldsymbol{x}(q, \omega)+\boldsymbol{d}(q, \omega),
$$

where $\boldsymbol{y}$ and $\boldsymbol{d}$ are column vectors of length $P, \boldsymbol{x}$ is a column vector of length $L$, and $\boldsymbol{H}$ is a matrix of size $P \times L$.

The wave-domain transforms after [39], [41] decompose the wave field on the 2D plane into the expansion coefficients with respect to circular harmonics [47], which are orthogonal basis functions on a circle. Therefore, the wave-domain transform for the error sensor signals is defined as,

$$
Y_{\mathrm{w}}^{m}(q, \omega)=\frac{1}{P} \sum_{p=1}^{P} Y_{p}(q, \omega) e^{-i m \phi_{p}}, \quad m=-M_{0}, \ldots, M_{0},
$$

where the subscript $(\cdot)_{\mathrm{w}}$ represents the wave-domain signal, $m$ is indexing the mode, and where the upper mode limit is given by $M_{0}=\left\lceil k r_{0}\right\rceil$ with the wave number $k=\omega / c$ and the microphone array radius $r_{0}$ [48]. Using (5), we transform the error signals into a finite number of wave-domain coefficients, i.e., $N_{m}=2 M_{0}+1$, which provides a compact form to describe the noise field, i.e., the superposition of the residual noise and auxiliary noise, within the control region.

The wave-domain transform for the secondary source signals is defined as the wave-domain signals produced by the loudspeakers within the control region under free-field conditions [38], [39], that is

$$
\begin{array}{r}
X_{\mathrm{w}}^{m}(q, \omega)=\frac{1}{P} \sum_{p=1}^{P} \sum_{\ell=1}^{L} G_{p \ell}(\omega) X_{\ell}(q, \omega) e^{-i m \phi_{p}}, \\
m=-M_{0}, \ldots, M_{0},
\end{array}
$$

where $G_{p \ell}(\omega)$ is the Green's function for the wave equation to represent the propagation of a $3 \mathrm{D}$ point source in the free field from the loudspeaker $\ell$ to the microphone $p$ [49].

We use $\boldsymbol{T}_{1}^{\mathrm{f}}$ and $\boldsymbol{T}_{2}^{\mathrm{f}}$ to denote the forward wave-domain transform for secondary source signals and error sensor signals, respectively, based on (5) and (6) [39], [50]. The backward wave-domain transforms $\boldsymbol{T}_{1}^{\mathrm{b}}$ and $\boldsymbol{T}_{2}^{\mathrm{b}}$ are defined simply as the inverse of the corresponding forward transform. Then, we obtain, equivalently to (3), in the wave domain,

$$
\boldsymbol{y}_{\mathrm{w}}(q, \omega)=\boldsymbol{H}_{\mathrm{w}}(q, \omega) \boldsymbol{x}_{\mathrm{w}}(q, \omega)+\boldsymbol{d}_{\mathrm{w}}(q, \omega),
$$

where for a given frequency $\omega, \boldsymbol{y}_{\mathrm{w}}=\boldsymbol{T}_{2}^{\mathrm{f}} \boldsymbol{y}, \boldsymbol{d}_{\mathrm{w}}=\boldsymbol{T}_{2}^{\mathrm{f}} d$, and $\boldsymbol{x}_{\mathrm{w}}=\boldsymbol{T}_{1}^{\mathrm{f}} \boldsymbol{x}$ are column vectors of length $N_{m}$ with elements $Y_{\mathrm{w}}^{m}(q, \omega), D_{\mathrm{w}}^{m}(q, \omega)$, and $X_{\mathrm{w}}^{m}(q, \omega)$, respectively. The wavedomain secondary path $\boldsymbol{H}_{\mathrm{w}}$ is a square matrix of size $N_{m} \times$ $N_{m}$ representing the coupling of the acoustic channels in the wave domain. In addition, we have the following conversion relationship [42],

$$
\boldsymbol{H}=\boldsymbol{T}_{2}^{\mathrm{b}} \boldsymbol{H}_{\mathrm{w}} \boldsymbol{T}_{1}^{\mathrm{f}}, \quad \boldsymbol{H}_{\mathrm{w}}=\boldsymbol{T}_{2}^{\mathrm{f}} \boldsymbol{H} \boldsymbol{T}_{1}^{\mathrm{b}} .
$$

Note that the loudspeaker and microphone numbers $L$ and $P$ are related to $N_{m}$ only by the upper mode limit $M_{0}$, i.e., $L \geq N_{m}$ and $P \geq N_{m}$ with $N_{m}=2 M_{0}+1$; hence, these numbers do not have to be identical. For simplicity of notation, we still assume $L=P=N_{m}$ in this work. Otherwise, at least one of the equations in (8) would require a pseudo inverse as an approximation for the inverse transform $\boldsymbol{T}_{1}^{\mathrm{b}}$ or $\boldsymbol{T}_{2}^{\mathrm{b}}$. 


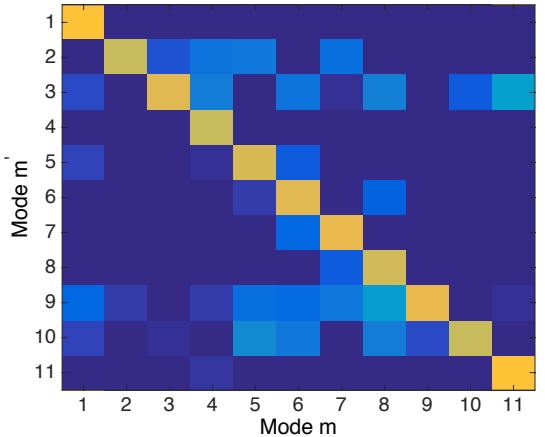

(a)

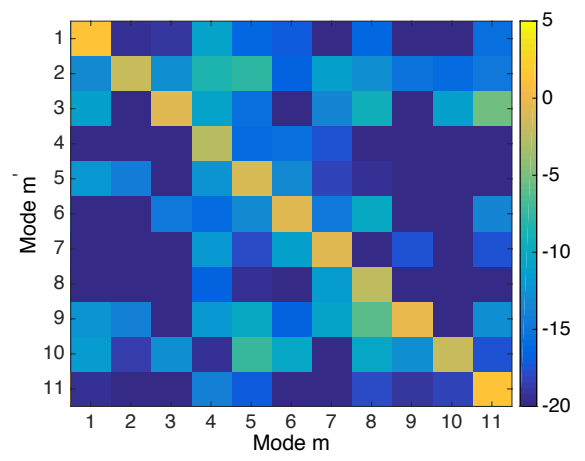

(b)

Fig. 2. Plots of the wave-domain secondary path $\boldsymbol{H}_{\mathrm{w}}$ in a $2 \mathrm{D}$ room of size $5 \mathrm{~m}$ $\times 7 \mathrm{~m}$ with reflection coefficient and reverberation time (a) $\beta=0.5, T_{60} \approx$ $145 \mathrm{~ms}$ and (b) $\beta=0.8, T_{60} \approx 300 \mathrm{~ms}$, respectively. The loudspeaker array and microphone array are concentrically placed as shown in Fig. 1.

\section{SECONDARY PATH MODELLING IN THE WAVE DOMAIN}

In this section, we show the characteristics of the wavedomain secondary path and a systematic approach to model it under three typical situations, i.e., i) concentric loudspeaker and microphone array placement, ii) non-concentric loudspeaker and microphone array placement, and iii) placement of loudspeakers and microphones with positioning errors.

\section{A. Diagonal Structure of $\boldsymbol{H}_{w}$}

A special property of the wave-domain secondary path $\boldsymbol{H}_{\mathrm{w}}$ as shown in Fig. 2 is that the matrix has dominant diagonal components [51], [52]. Especially, in mildly to moderately reverberant environments and with omnidirectional loudspeakers/microphones, the matrix $\boldsymbol{H}_{\mathrm{w}}$ can be approximated as a diagonal matrix when the loudspeaker array and microphone array are concentrically placed [39], [53]. A special case is for free-field sound propagation and ideal transducers, where $\boldsymbol{H}_{\mathrm{w}}$ is an identity matrix $\boldsymbol{I}$.

A more general representation of $\boldsymbol{H}_{\mathrm{w}}$ can be written as,

$$
\boldsymbol{H}_{\mathrm{w}}=\boldsymbol{I}+\boldsymbol{\Gamma}
$$

where $\boldsymbol{I}$ represents the direct-path components and $\boldsymbol{\Gamma}$ represents only the reverberant part relative to the direct-path part in the wave domain. In a moderately reverberant environment, the non-diagonal entries of $\boldsymbol{\Gamma}$ have values much smaller

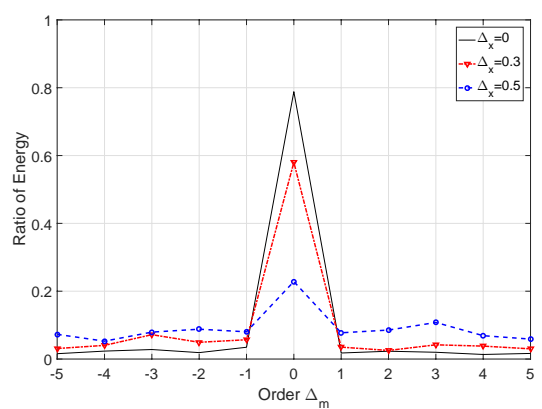

(a)

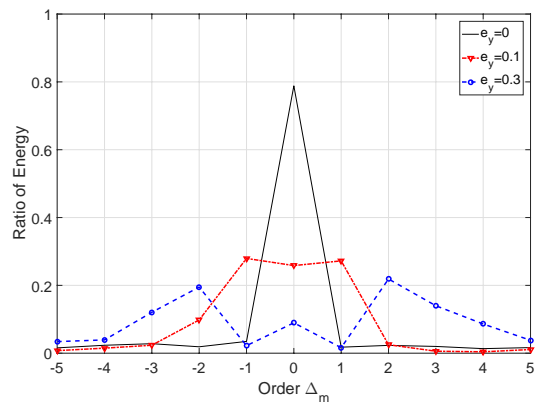

(b)

Fig. 3. The ratio of energy $E_{\Delta_{m}}$ of the wave-domain secondary path $\boldsymbol{H}_{\mathrm{w}}$ at (a) different distances between the loudspeaker array center and microphone array center $\Delta_{x}$ and (b) different positioning errors of the loudspeaker array in a reverberant room with reflection coefficient $\beta=0.7$ and reverberation time $T_{60} \approx 210 \mathrm{~ms}$.

than 1 and thus $\boldsymbol{I}$ becomes the dominant part of the matrix $\boldsymbol{H}_{\mathrm{w}}$. This diagonal structure holds as long as reverberation remains weaker than the direct-path component, i.e., when the loudspeaker array is not too close to a wall, or the distance between the loudspeakers and microphones is not too large.

In the following, we investigate the influence of array positions on the wave-domain secondary path $\boldsymbol{H}_{\mathrm{w}}$. Two conditions are explored. The first case is that the loudspeaker array and microphone array are not concentrically placed, where the distance between two arrays $\Delta_{x}$ with respect to the $x$-axis is known as a prior and taken into account in the wavedomain transform. The second case is that there are additional positioning errors of the loudspeaker and microphone array $e_{y}$ which are unknown and not considered in the wave-domain transform. This resembles the setups in [39] and allows here a precise investigation of the origins of diagonal dominance without measurement errors, as the following investigations employ an image source model to simulate the acoustic wave fields for truly omnidirectional transducers and also without any positioning errors.

We investigate the energy captured in the off-diagonal components relative to the total energy in $\boldsymbol{H}_{\mathrm{w}}$, i.e., the ratio

$E_{\Delta_{m}}=\frac{\sum_{m=1}^{N_{m}}\left\|\boldsymbol{H}_{\mathrm{w}}\left(m, m_{\Delta_{m}}\right)\right\|^{2}}{\sum_{m=1}^{N_{m}} \sum_{m^{\prime}=1}^{N_{m}}\left\|\boldsymbol{H}_{\mathrm{w}}\left(m, m^{\prime}\right)\right\|^{2}},-M_{0} \leq \Delta_{m} \leq M_{0}$,

where $\Delta_{m}$ denotes the off-diagonal order. Thus, $\Delta_{m}=0$ corresponds to the main diagonal of $\boldsymbol{H}_{\mathrm{w}}$, and $\Delta_{m}=1$, for 
instance, corresponds to the first minor diagonal above or right of the main diagonal, consisting of the elements $\boldsymbol{H}_{\mathrm{w}}(m, m+$ 1). Similarly, negative values of $\Delta_{m}$ correspond to the leftsided terms. As $\left|\Delta_{m}\right|$ increases, the off-diagonal terms are further away from the diagonal components.

Given $\boldsymbol{H}_{\mathrm{w}}$ is a square matrix of size $N_{m} \times N_{m}$, for the term $\boldsymbol{H}_{\mathrm{w}}\left(m, m_{\Delta_{m}}\right)$, we have $1 \leq m \leq N_{m}$ and

$$
m_{\Delta_{m}}= \begin{cases}N_{m} & \text { if } \bmod \left(m+\Delta_{m}+N_{m}\right. \\ & \left.N_{m}\right)=0 \\ \bmod \left(m+\Delta_{m}+N_{m}, N_{m}\right) & \text { otherwise }\end{cases}
$$

Furthermore, given the structure of the matrix $\boldsymbol{H}_{\mathrm{w}}$ as shown in Fig. 2, we consider a cyclic wrapping of the minor diagonals, i.e., including the two-sided terms of the main diagonal, for computing energy ratios according to (10).

Figure 3 shows an example for the ratio of energy captured in the diagonal components $E_{0}$ and that in the off-diagonal components $E_{\Delta_{m}}$ at different displacement distances between the loudspeaker array centre and microphone array centre, i.e., $\Delta_{x} \in\{0,0.3,0.5\} \mathrm{m}$, and at different positioning errors of the loudspeaker array, i.e., $e_{y} \in\{0,0.1,0.3\} \mathrm{m}$. We also investigated $\boldsymbol{H}_{\mathrm{w}}$ for different room sizes, room acoustics, and array configurations and observed similar results.

Overall, the structure of $\boldsymbol{H}_{\mathrm{w}}$ demonstrates unique properties, which can be summarized as follows:

- The diagonal dominance reduces with increasing distance between the loudspeaker array and microphone array centre $\Delta_{x}$ and increasing positioning error $e_{y}$.

- When the displacement $\Delta_{x}$ is taken into account in the wave-domain transform, the property of diagonal dominance still exists as shown in Fig. 3 (a). However, an increased displacement of the array centres increases the energy in the off-diagonals in an unpredictable way. So, Fig. 3 (a) does not provide conclusive knowledge on which off-diagonals may be important in case of perfectly known geometry.

- In the second case Fig. 3 (b), where the additional positioning errors $e_{y}$ are not accounted for in the wavedomain transform, the off-diagonal terms become more important. However, the off-diagonals' influences decrease with the order $\Delta_{m}$.

The above displacement dependency of the diagonal structure has also been investigated in [38], [39] in the context of acoustic echo cancellation and room equalization.

\section{B. Wave-domain secondary path model}

As pointed out in Sec. III-A, for mildly to moderately reverberant environments, the wave-domain SPM can be approximated as a diagonal matrix, no matter if the loudspeaker array and the microphone array are placed concentrically or not. However, additional off-diagonal components should be included for strong reverberation and also for improving the robustness against the loudspeaker/microphone positioning error. Therefore, we do not restrict the wave-domain model for ANC to a single diagonal, as done in the initial publications [40], [51], [52]. Instead, we propose to use the generalized wave-domain model from [38] as SPM for ANC.

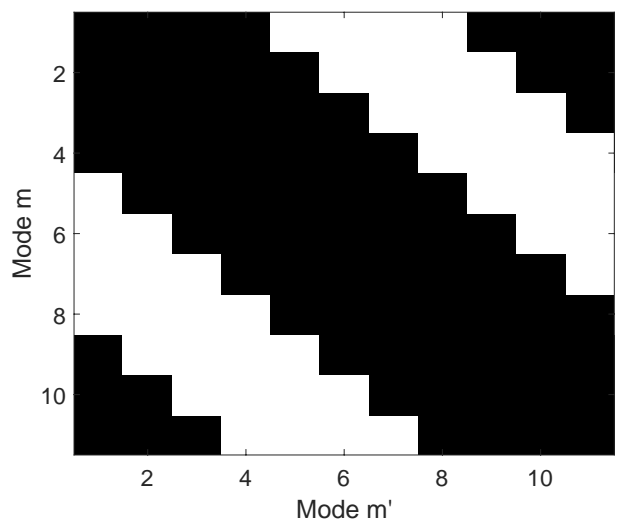

Fig. 4. A plot of the weighting matrix $C$ with $M_{0}=5, N_{m}=11$ and $m_{0}=3$, where black denotes the value of 1 and white denotes the value of 0.

The proposed wave-domain SPM has a binary weighting matrix $C$ applied to the full wave-domain SPM, that is

$$
\tilde{\boldsymbol{H}}_{\mathrm{w}}=\boldsymbol{C} \circ \boldsymbol{H}_{\mathrm{w}},
$$

where $\circ$ represents the Hadamard product (or element-wise product) of two matrices and the elements of the constraint matrix $C$ are chosen as

$$
\boldsymbol{C}_{m, m^{\prime}}= \begin{cases}1 & \left|m-m^{\prime}\right| \leq m_{0} \text { or } \\ & \bmod \left(-\left|m-m^{\prime}\right|, N_{m}\right) \leq m_{0} \\ 0 & \text { otherwise }\end{cases}
$$

with $1 \leq m, m^{\prime} \leq N_{M}$ and the upper limit of the weighting order $m_{0} \leq M_{0}$. Figure 4 shows a plot of the matrix $\boldsymbol{C}$ with $M_{0}=5, N_{m}=11$ and $m_{0}=3$. Note that the higher the order $m_{0}$, the more off-diagonal components for the wave-domain SPM $\boldsymbol{H}_{\mathrm{w}}$ are incorporated into the model.

Wave-domain processing has successfully been used for acoustic echo cancellation [36], large room equalization [37], [38], [52], and ANC [11], [18], [22] applications; next we will make use of it to develop a wave-domain ANC with online SPM.

\section{Proposed waVe-domain ANC With online SPM}

\section{A. Adaptive algorithm}

The block diagram of the proposed wave-domain ANC with online SPM is shown in Fig. 5. Referring to (2), there are two parts of the secondary source signals, $\boldsymbol{x}_{\mathrm{w}}^{\mathrm{c}}(q)$ and $\boldsymbol{x}_{\mathrm{w}}^{\mathrm{e}}(q)$ for ANC and SPM, respectively. The received error signals in the wave domain are represented as

$$
\boldsymbol{y}_{\mathrm{w}}=\boldsymbol{H}_{\mathrm{w}}\left(\boldsymbol{x}_{\mathrm{w}}^{\mathrm{c}}+\boldsymbol{x}_{\mathrm{w}}^{\mathrm{e}}\right)+\boldsymbol{d}_{\mathrm{w}} .
$$

The design of the proposed system is based on minimizing the following NLMS-type cost function,

$$
\begin{aligned}
\mathcal{J} & =\left\|\boldsymbol{y}_{\mathrm{w}}-\hat{\boldsymbol{H}}_{\mathrm{w}} \boldsymbol{x}_{\mathrm{w}}^{\mathrm{e}}\right\|^{2} \\
& =\|\underbrace{\boldsymbol{d}_{\mathrm{w}}+\boldsymbol{H}_{\mathrm{w}} \boldsymbol{x}_{\mathrm{w}}^{\mathrm{c}}}_{\text {ANC }}+\underbrace{\left(\boldsymbol{H}_{\mathrm{w}}-\hat{\boldsymbol{H}}_{\mathrm{w}}\right) \boldsymbol{x}_{\mathrm{w}}^{\mathrm{e}}}_{\text {SPM }}\|^{2} .
\end{aligned}
$$

where $\hat{\boldsymbol{H}}_{\mathrm{w}}$ represents an estimate of the wave-domain secondary path. Note that $\boldsymbol{H}_{\mathrm{w}}$ is a fully populated square matrix, 


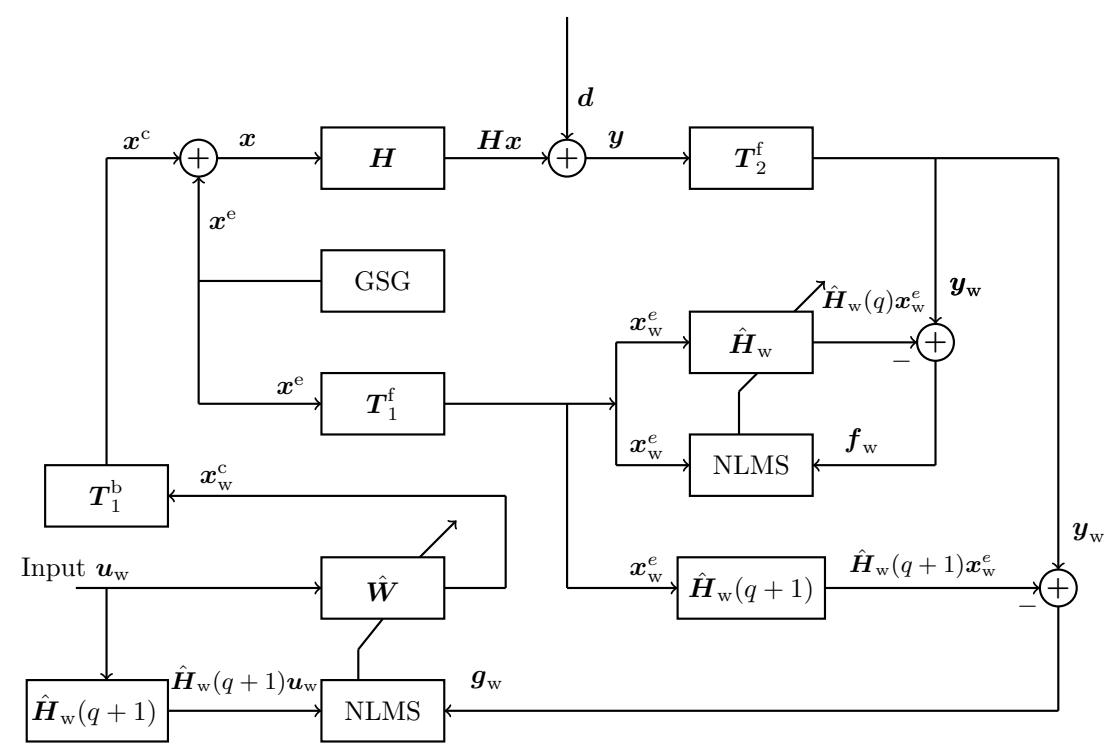

Fig. 5. Block diagram of wave-domain ANC with online SPM (see [40] for a corresponding system with single-diagonal SPM).

whereas $\hat{\boldsymbol{H}}_{\mathrm{w}}$ is an estimate for $\tilde{\boldsymbol{H}}_{\mathrm{w}}$ from Sec. III-B and is accordingly a sparsely populated matrix with a few diagonal elements only and cyclically extended minor diagonals.

In the following, adaptive algorithms are applied to deal with non-stationary noise and time-varying acoustic environments. Based on the proposed wave-domain SPM, the update for online estimation is performed using the following NLMStype equation

$$
\hat{\boldsymbol{H}}_{\mathrm{w}}(q+1)=\boldsymbol{C} \circ\left(\hat{\boldsymbol{H}}_{\mathrm{w}}(q)+\mu_{\mathrm{e}} \frac{\boldsymbol{f}_{\mathrm{w}}(q) \overline{\boldsymbol{x}_{\mathrm{w}}^{\mathrm{e}}(q)}}{\left\|\boldsymbol{x}_{\mathrm{w}}^{\mathrm{e}}(q)\right\|^{2}+\delta_{e}}\right),
$$

where the time block index $q$ denotes the iteration, $\overline{(\cdot)}$ denotes the conjugate transpose, and the a priori error

$$
\begin{aligned}
\boldsymbol{f}_{\mathrm{w}}(q) & =\boldsymbol{y}_{\mathrm{w}}(q)-\hat{\boldsymbol{H}}_{\mathrm{w}}(q) \boldsymbol{x}_{\mathrm{w}}^{\mathrm{e}}(q) \\
& =\left(\boldsymbol{H}_{\mathrm{w}}-\hat{\boldsymbol{H}}_{\mathrm{w}}(q)\right) \boldsymbol{x}_{\mathrm{w}}^{\mathrm{e}}(q)+\underbrace{\boldsymbol{d}_{\mathrm{w}}+\boldsymbol{H}_{\mathrm{w}} \boldsymbol{x}_{\mathrm{w}}^{\mathrm{c}}(q)}_{\text {interference due to residual noise }} .
\end{aligned}
$$

The auxiliary noise $\boldsymbol{x}^{e}$ is produced using the Gold sequence generator (GSG) as detailed in Sec. IV-B.

A wave-domain adaptive filter is used for generating antinoise signals, that is

$$
\boldsymbol{x}_{\mathrm{w}}^{\mathrm{c}}(q+1)=\hat{\boldsymbol{W}}(q+1) \boldsymbol{u}_{\mathrm{w}}(q),
$$

where $\boldsymbol{u}_{\mathrm{w}}(q)$ is an $N_{m}$ element column vector containing real white Gaussian noise. The NLMS algorithm is used to update the filter coefficients,

$$
\hat{\boldsymbol{W}}(q+1)=\hat{\boldsymbol{W}}(q)-\mu_{\mathrm{c}} \frac{\overline{\hat{\boldsymbol{H}}_{\mathrm{w}}(q+1)} \boldsymbol{g}_{\mathrm{w}}(q) \boldsymbol{u}_{\mathrm{w}}^{T}(q)}{\left\|\hat{\boldsymbol{H}}_{\mathrm{w}}(q+1)\right\|^{2}\left\|\boldsymbol{u}_{\mathrm{w}}(q)\right\|^{2}+\delta_{c}},
$$

where $(\cdot)^{T}$ denotes the transpose, with the a posteriori error

$$
\begin{aligned}
& \boldsymbol{g}_{\mathrm{w}}(q)=\boldsymbol{y}_{\mathrm{w}}(q)-\hat{\boldsymbol{H}}_{\mathrm{w}}(q+1) \boldsymbol{x}_{\mathrm{w}}^{\mathrm{e}}(q) \\
& =\underbrace{\left(\boldsymbol{H}_{\mathrm{w}}-\hat{\boldsymbol{H}}_{\mathrm{w}}(q+1)\right) \boldsymbol{x}_{\mathrm{w}}^{\mathrm{e}}(q)}_{\text {interference due to SPM error }}+\boldsymbol{d}_{\mathrm{w}}+\boldsymbol{H}_{\mathrm{w}} \boldsymbol{x}_{\mathrm{w}}^{\mathrm{c}}(q) .
\end{aligned}
$$

The step sizes $\left(\mu_{e}\right.$ and $\left.\mu_{c}\right)$ and regularization terms $\left(\delta_{e}\right.$ and $\delta_{c}$ ) are important parameters to tune for a stable system with fast adaptation performance and sufficient modelling accuracy. Using a variable step size can further improve the system flexibility and stability, which however is not in the scope of this work.

\section{B. Auxiliary Noise}

The two adaptation processes in the proposed system influence each other due to the two interference terms. The signal properties of the auxiliary noise $\boldsymbol{x}^{\mathrm{e}}(q)$ have great influence on the system performance. Here, we use a set of Gold sequences [54] that have extremely small crosscorrelation within the set to generate $\boldsymbol{x}^{\mathrm{e}}(q)$ for online SPM. As the system is designed to cancel tonal noise, i.e., a singlefrequency noise or multiple-frequency noise, the auxiliary noise is generated using the ASK (Amplitude Shift Keying) modulation technique, i.e., during the time block $q$ or $\boldsymbol{t}_{q}=$ $\left[t_{q}(0), \ldots, t_{q}(N-1)\right]^{T}$

$$
\begin{aligned}
x_{\ell}^{e}(q) & =g_{s ; \ell}(q) \cos \left(2 \pi f_{d} \boldsymbol{t}_{q}\right) \\
& =g_{s ; \ell}(q)\left[\cos \left(2 \pi f_{d} t_{q}(0)\right), \ldots, \cos \left(2 \pi f_{d} t_{q}(N-1)\right)\right]^{T}
\end{aligned}
$$

with the carrier frequency being the same as the noise frequency $f_{d}$ and the binary amplitude assigned by the Gold sequence generator (GSG) $g_{s ; \ell}(q)$. Using this notation, the auxiliary noise in the frequency domain and wave domain are referred to by column vectors, $\boldsymbol{x}^{e}(q)=\left[g_{s ; 1}^{e}(q), \ldots, g_{s ; L}^{e}(q)\right]^{T}$ and $\boldsymbol{x}_{\mathrm{w}}^{e}(q)=\boldsymbol{T}_{1}^{\mathrm{f}} \boldsymbol{x}^{e}(q)$, respectively. As shown in the experimental section, using such a sequence would only require an additional $\sim 10 \%$ noise level increase during the online operation of SPM. A better strategy is to adaptively reduce the auxiliary noise along the residual of the primary noise. Thereby, one should be able to minimize the auxiliary noise, while even accounting for perceptual criteria if desired. This will be one of the aims of future work. 
TABLE I

COMPUTATIONAL COMPLEXITY OF THE WAVE-DOMAIN ANC COMPARED WITH THE FREQUENCY-DOMAIN AND TIME-DOMAIN MULTICHANNEL ANC.

\begin{tabular}{ll}
\hline Method & No. of multiplications per time block \\
\hline Wave-domain ANC & $\left.\mathcal{O}\left(N_{f} \log N_{f}(L+P)\right)+\mathcal{O}\left(N_{m}(L+P)+N_{m}^{2}\left(2 \Delta_{m}+1\right)\right)\right)$ \\
Frequency-domain ANC & $\mathcal{O}\left(N_{f} \log N_{f}(L+P)\right)+\mathcal{O}(L P)$ \\
Time-domain ANC & $\mathcal{O}\left(N_{f} L P\right)$ \\
\hline
\end{tabular}

\section{Computational Complexity}

The computational complexity analysis of the method described in this paper is based on the number of multiplications per iteration or time block. A comparison between the proposed wave-domain ANC, the frequency-domain and timedomain multichannel ANC is presented in Table 1 using the big $\mathcal{O}$ notation. Here $N_{f}$ is the tap-weight length of the ANC filter (assuming the same for the online SPM filter and FFT operation); $L$ and $P$ denote the number of secondary sources and error sensors, respectively; $N_{m}$ represents the total number of modes used in the wave-domain ANC.

In the multichannel setup, the time-domain ANC has the largest computational complexity as it is proportional to the multiplication of the filter length, the number of secondary sources and that of error sensors. The computational complexity of the wave-domain ANC and frequency-domain ANC, on the other hand, is determined by two parts, the FFT operation $\mathcal{O}\left(N_{f} \log N_{f}(L+P)\right)$ and the two-step adaptive processing. The latter part for the wave-domain ANC consists of the wave-domain transform $\mathcal{O}\left(N_{m}(L+P)\right)$ and wavedomain processing $\mathcal{O}\left(N_{m}^{2}\left(2 \Delta_{m}+1\right)\right)$. The wave-domain ANC and frequency-domain ANC systems have roughly the same computational complexity while the latter has a faster convergence performance. Only when a massive array setup is deployed (i.e., $N_{m} \ll L, P$ ) or a diagonal-dominant SPM is adopted (i.e., $\Delta_{m}=0$ or 1 ), the wave-domain ANC has the smallest computational complexity. On the other hand, the wave-domain ANC and frequency-domain ANC are based on block processing and thus have some processing lag while the time-domain ANC has a zero-processing lag.

\section{Evaluation}

\section{A. Simulation Setup}

The evaluations are performed in a simulated room environment which is generated using the image-source model [55]. The geometry of the experimental setup is shown in Fig. 6, where the room has a size of $5 \mathrm{~m} \times 7 \mathrm{~m}$ and consists of four planar walls with the reflection coefficient $\beta$ and perfectlyabsorbing floor and ceiling. An 11-element loudspeaker array (blue) and an 11-element microphone array (red) with radii of $1.5 \mathrm{~m}$ and $0.5 \mathrm{~m}$, respectively, are concentrically placed at the point $(-0.5,0.5) \mathrm{m}$ relative to the room centre and used as the secondary sources and error sensors, respectively ${ }^{4}$. This corresponds to the optimal condition. In addition, we evaluate the robustness of the proposed system under different kinds of array displacement, including i) the loudspeaker array and

\footnotetext{
${ }^{4}$ The number of loudspeakers and microphones are determined to satisfy the dimensionality requirement as shown in (5) and (6), that is given $M_{0}$ both $L$ and $P$ should be at least $2 M_{0}+1$.
}

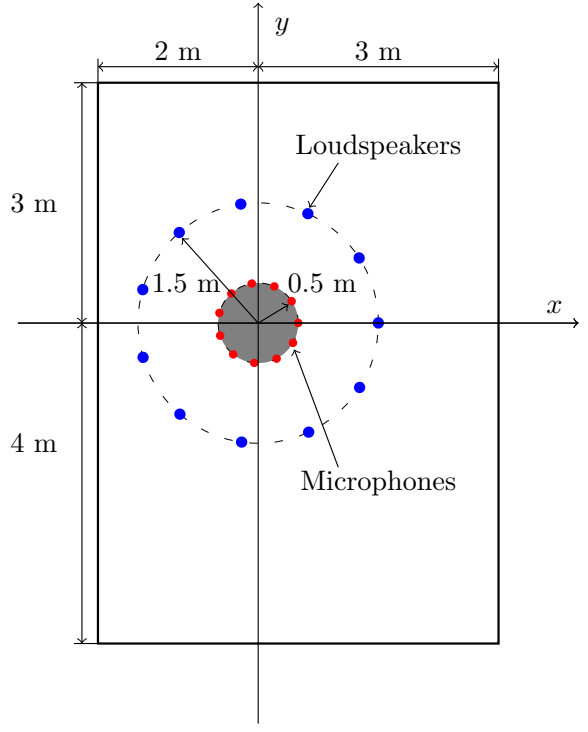

Fig. 6. Simulation setup for evaluation of the wave-domain ANC in a reverberant room. Uniform circular arrays of loudspeakers (blue) and microphones (red) are placed on a 2D plane for noise cancellation within the shaded region.

microphone array are not concentrically placed and ii) the loudspeaker array is placed with additional positioning errors. The control of a single-frequency noise and multi-frequency noise are examined in the following, where the loudspeakers and noise source are modelled as 3D point sources. A sampling rate of $8 \mathrm{kHz}$ and time frames of length 2048 samples are employed. Furthermore, microphone recordings with $40 \mathrm{~dB}$ SNR are assumed.

The system is evaluated by two objective measures, the SPM error and the noise reduction performance. The SPM error refers to the error between the estimated and true channel of the multiple-loudspeaker-multiple-microphone setup, that is

$$
e_{\text {spm }}(q)=10 \times \log 10 \frac{\|\boldsymbol{H}-\hat{\boldsymbol{H}}(q)\|^{2}}{\|\boldsymbol{H}\|^{2}} .
$$

Noise reduction performance is investigated at the microphone positions as well as within the control region of a spatially extended 2D plane, i.e.,

$$
\begin{aligned}
& e_{\mathrm{anc}}^{\mathrm{m}}(q)=10 \times \log 10 \frac{\sum_{p=1}^{P}\left\|\boldsymbol{r}_{\mathrm{anc}}(p, q)\right\|^{2}}{\sum_{p=1}^{P}\|\boldsymbol{d}(p)\|^{2}} \\
& e_{\mathrm{anc}}^{\mathrm{s}}(q)=10 \times \log 10 \frac{\int_{\Omega}\left\|\boldsymbol{r}_{\mathrm{anc}}(\boldsymbol{y}, q)\right\|^{2} d \boldsymbol{y}}{\int_{\Omega}\|\boldsymbol{d}(\boldsymbol{y})\|^{2} d \boldsymbol{y}},
\end{aligned}
$$

where $\boldsymbol{r}_{\mathrm{anc}}(p, q), \boldsymbol{r}_{\mathrm{anc}}(\boldsymbol{y}, q)$ represents the residual signal at the $p$ th microphone or a point $\boldsymbol{y}$ within the control region $\Omega$ at the $q$ th iteration; $\boldsymbol{d}(p), \boldsymbol{d}(\boldsymbol{y})$ denotes the corresponding 


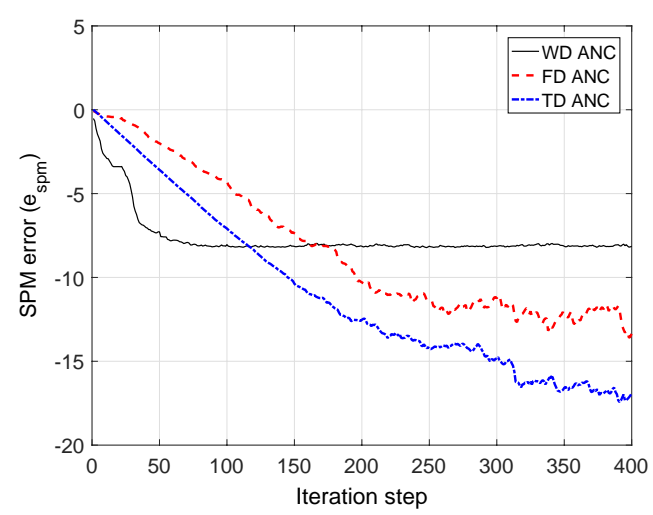

(a)

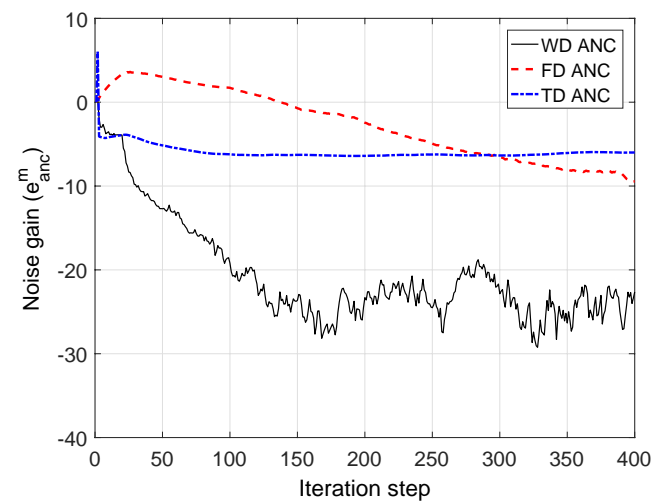

(b)

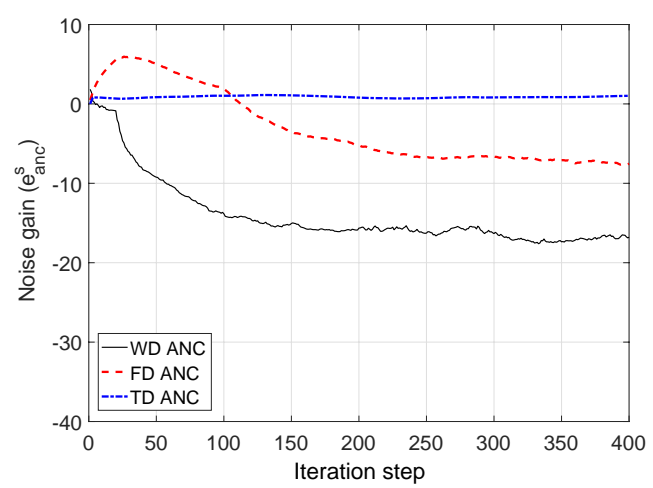

(c)

Fig. 7. Adaptation performance of the proposed WD ANC compared with the conventional FD ANC and TD ANC. Plots correspond to (a) online SPM error and noise reduction at (b) the microphone positions or (c) within the 2D control region. The auxiliary noise injected into the control region for online SPM is $1 \mathrm{~dB}$ lower than the primary noise level.

primary noise. In the simulation, (24) is evaluated from 1296 sampling points uniformly arranged in the control region.

\section{B. Single Source and Single Frequency Noise Field}

In the first experiment, active control of a single-frequency noise using multichannel ANC is evaluated. The primary noise source is located at $r=2 \mathrm{~m}, \phi=45^{\circ}$ with respect to the array centre and operates at a frequency of $500 \mathrm{~Hz}$.

1) Optimal condition: The optimal condition corresponds to the case that the loudspeaker array and microphone array

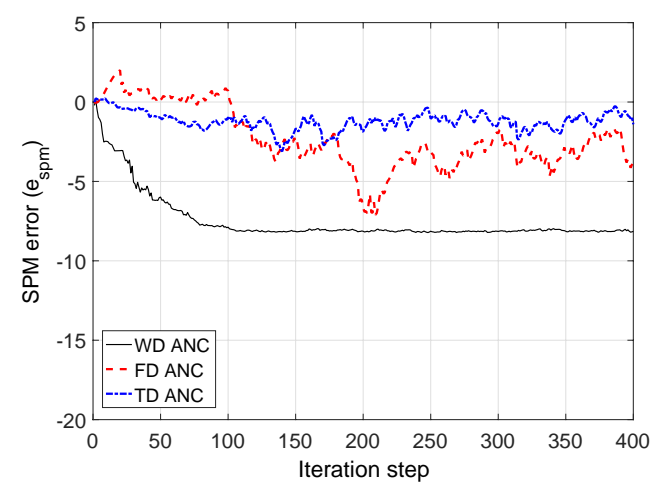

(a)

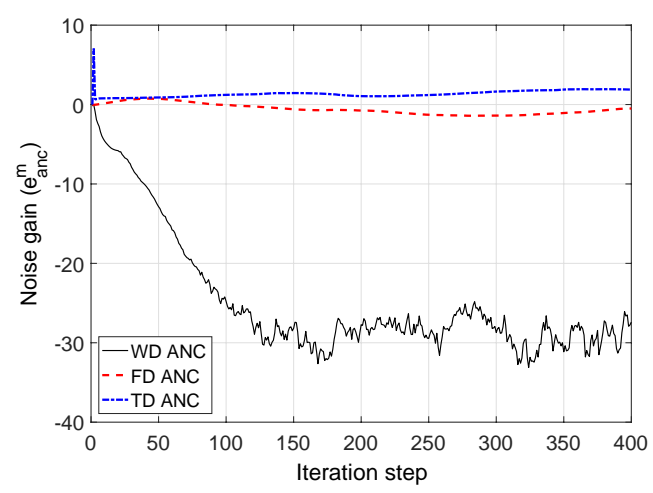

(b)

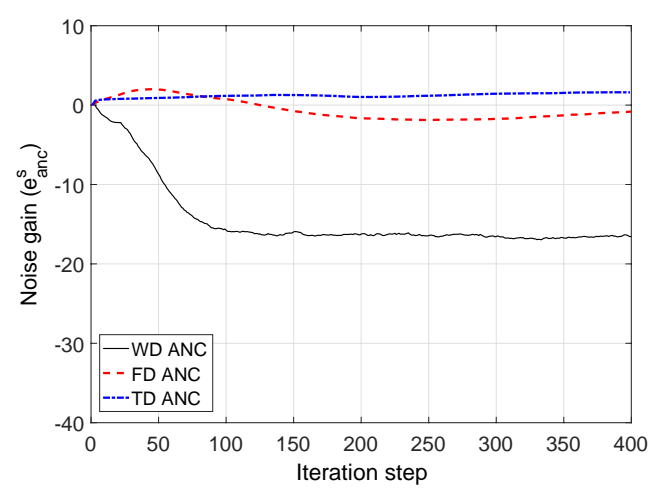

(c)

Fig. 8. Adaptation performance of the proposed WD ANC compared with the conventional FD ANC and TD ANC. Plots correspond to (a) online SPM error and noise reduction at (b) the microphone positions or (c) within the 2D control region. The auxiliary noise injected into the control region for online SPM is $10 \mathrm{~dB}$ lower than the primary noise level.

are concentrically placed. In this case we model the SPM $\hat{\boldsymbol{H}}_{\mathrm{w}}$ by a diagonal matrix and thereby also neglect reflections on the walls of the room. Then, the proposed wave-domain ANC system works as a single-mode adaptation (each mode is adapted independently from the others). The performance of the proposed system is demonstrated through comparison with the frequency-domain (FD) and time-domain (TD) multichannel ANC systems.

In the first experiment, the room is simulated with the reverberation coefficient $\beta=0.6$, reflection order 12 , and 
reverberation time $T_{60} \approx 170 \mathrm{~ms}$. The adaptation performance of the proposed wave-domain (WD) ANC system is plotted in Figs. 7 and 8, in comparison with the feedback FD ANC system [2] and the feed-forward TD ANC system [34]. In these three systems, the NLMS adaptation is adopted with the step size $\mu_{e}=1, \mu_{c}=1$ in WD ANC, $\mu_{e}=0.01, \mu_{c}=0.1$ in FD ANC, and $\mu_{e}=0.1, \mu_{c}=0.001$ in TD ANC. These values are chosen for the fastest convergence and a stable performance of each system.

Plots in Fig. 7 and Fig. 8 show the system performance with different levels of auxiliary noise injected for online SPM. In Fig. 7, the auxiliary noise level is around $1 \mathrm{~dB}$ lower than the primary noise level. Note that here we are dealing with a large array setup (11 loudspeakers $\times 11$ microphones). This is the least amount of auxiliary noise required for online SPM of the FD ANC system to work. The feed-forward TD system, given a reference of the original noise, achieves the lowest online SPM error. However, its noise reduction performance is the worst. This can be explained by the fact that the TD system has a feed-forward structure and, thus, relies on a high coherence between the reference and error signals. The reverberation used in our simulations causes this coherence to be rather low, which ultimately limits the noise reduction performance for TD. In contrast, the WD and FD approaches are realized as a feedback structure, i.e., they do not rely on a reference signal and suffer from a possibly low coherence. The FD approach converges slowly but after about 3000 iterations, it achieves the same noise reduction performance at the microphone positions as the WD approach does. The large auxiliary noise levels used for this simulation, however, are often unacceptable for human listeners which then rules out the practical use of FD ANC and TD ANC. The proposed WD ANC system demonstrates the most stable adaptation performance at error microphone positions and also within the control region.

In Fig. 8, the auxiliary noise level is reduced to a very low level, which is around $10 \mathrm{~dB}$ lower than the primary noise level. The proposed WD ANC converges after around 100 iterations while the FD ANC and TD ANC do not achieve any noise suppression. In addition, comparing Fig. 7 (b), (c) to Fig. 8 (b), (c), a higher noise reduction is observed using WD ANC with lower level auxiliary noise. This can be explained as follows: As expected, the auxiliary noise level should be high enough for an accurate estimation of the SPM during its online operation. On the one hand, the required auxiliary noise level increases with the number of non-zero elements in the wave-domain SPM to be estimated. For example, when modelling the wave-domain secondary path as a diagonal matrix (i.e., a single-mode adaptation, or each mode is adapted independently), the smallest auxiliary noise level is required. On the other hand, an excessive amount of auxiliary noise interferes with the ANC adaptation and thus results in a larger ANC error (or degraded noise reduction performance). Therefore, there is a trade-off between the accuracy of online SPM and the ANC noise reduction performance through an appropriate choice of the auxiliary noise level. The results here show that when the online SPM is converged, the noise reduction performance of the wave-domain ANC is further improved with a low-level auxiliary noise. These results show a clear advantage of the wave-domain ANC.

In both examples, using the proposed system the SPM error is limited to $-8.21 \mathrm{~dB}$. This modelling error is mainly caused by not incorporating off-diagonal terms in $\hat{\boldsymbol{H}}_{\mathrm{w}}$, and not modelling the room reflections; however, a significant amount of noise reduction is still achieved. Note that the SPM error evaluated in simulations refers to the error between the estimated and the true channel of the multiple-loudspeakermultiple-microphone setup. Based on (22), the SPM accuracy using the proposed WD approach is low; however, the diagonal-dominant components of the secondary path in the model $\tilde{\boldsymbol{H}}_{\mathrm{w}}$ are estimated with much higher accuracy (i.e., error less than $-20 \mathrm{~dB}$ ). As only those modelled SP components are considered in the proposed ANC system which are also estimated accurately, the WD approach can achieve the desired noise reduction. In the current setup, the critical distance is about $1 \mathrm{~m}$, roughly the same as the difference between the radius of the loudspeaker array and the microphone array. The results with the full matrix of the wave-domain SPM is investigated in the following.

We further investigate the performance of the proposed WD ANC with different values of the upper mode limit $m_{0}$, where $m_{0}=1$ and $m_{0}=5$ correspond to modelling the wavedomain SP as a diagonal matrix and a full matrix, respectively. The results in Fig. 9 show that, as $m_{0}$ increases, the SPM error reduces as more wave-domain coefficients are incorporated into the model; however, the noise reduction performance is not improved significantly and its convergence performance degrades. Especially, when the wave-domain SP is modelled as a full matrix, the WD approach has roughly the same noise reduction performance as the FD approach (the red dashed lines in Fig. 7 (b) and (c)). The auxiliary noise level also increases with the order $m_{0}$ as more coefficients are required to be estimated. These results confirm that modelling the SP as a diagonal matrix is the most effective strategy for the optimal condition.

For the setup evaluated in Fig. 8, Fig. 10 (a) plots the sound field generated by the proposed system after the system converges. As can be seen, a quiet zone is produced within the control region surrounded by the microphones. The recordings of the original noise, auxiliary noise for SPM, and residual primary noise measured at microphone $1\left(r_{1}=0.5 \mathrm{~m}, \phi_{1}=0\right.$ w.r.t. the array center) in this example are plotted in Fig. 10 (b). A comparison among these recordings shows that the required auxiliary noise level is relatively low. The proposed system shows a long convergence time. This is due to the fact that it performs spatial ANC and online SPM simultaneously while the secondary path in a reverberant room between a loudspeaker and a microphone usually has a very long impulse response (i.e., from several hundred to several thousand taps). This means that when we implement block-adaptive filters to transform the signals from the time domain to the frequency domain and thereafter to the wave domain, a sufficiently long block size to capture the entire secondary path is required. Thus, it is clear that the increased room reverberation leads to an increased convergence time. Note that most existing works on multichannel ANC assume that the secondary path is known as a prior [6], [7], [8], [11], [17], [18]. The only 


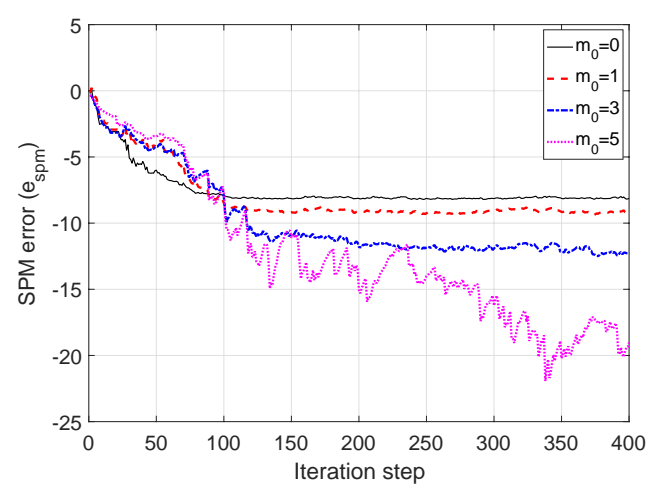

(a)

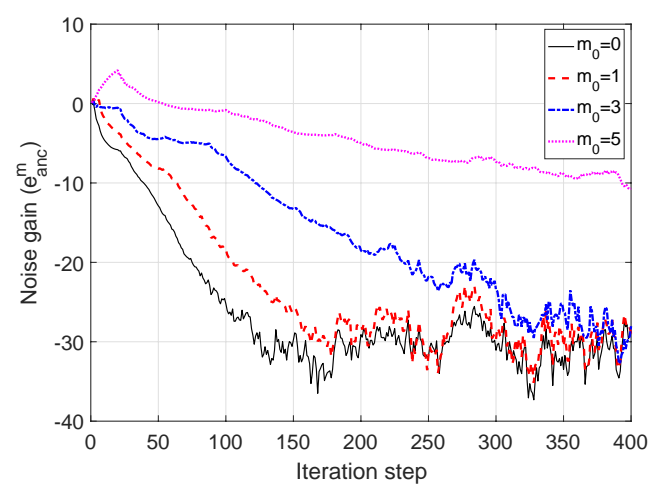

(b)

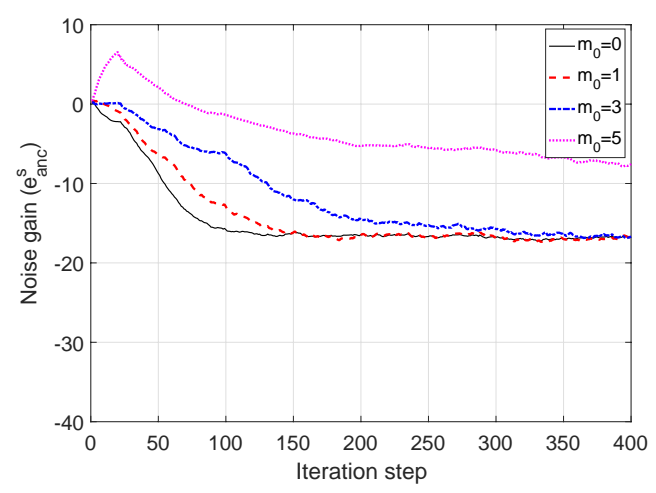

(c)

Fig. 9. Adaptation performance of the proposed WD ANC with different values of the upper mode limit $m_{0}$. Plots correspond to (a) online SPM error and noise reduction at (b) the microphone positions or (c) within the $2 \mathrm{D}$ control region.

known work on multichannel ANC with online SPM in [34] investigated a $1 \times 2 \times 2$ ANC system, i.e., only two secondary sources, two error microphones and one reference microphone are used.

As further results, the plots in Fig. 10 correspond to the case that a fixed low level of auxiliary noise is used for online SPM. For practical implementation, a better strategy is to produce the auxiliary noise which is proportional to the power of the residual signal. That is the level of the auxiliary noise can be decreased after a good ANC performance is achieved. As mentioned above, according adaptation strategies will be

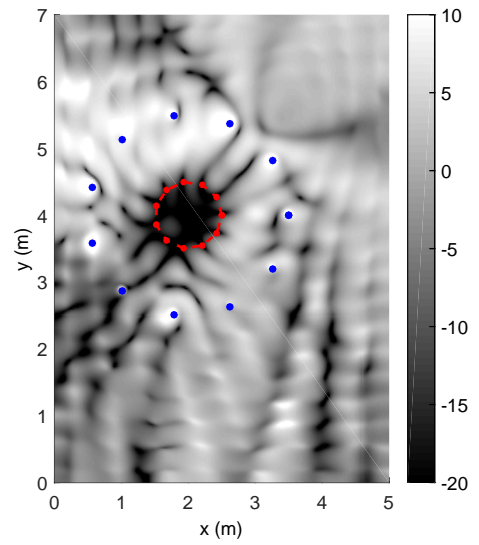

(a)
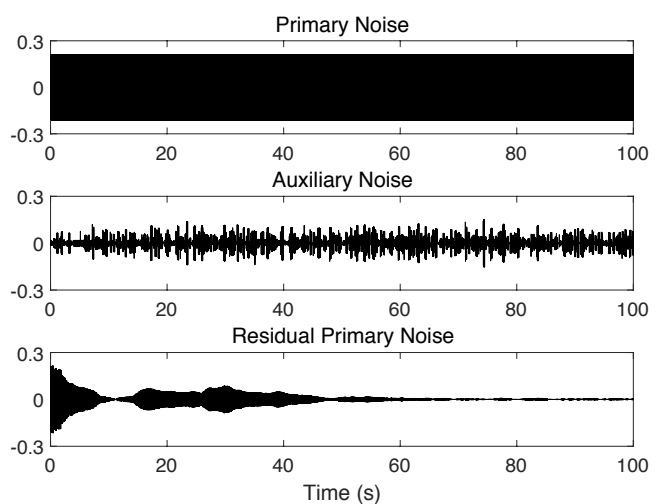

(b)

Fig. 10. Plots are (a) the sound field generated by the proposed WD ANC after the system converges and (b) a comparison of the original noise, auxiliary noise for SPM, and residual primary noise measured at one microphone.
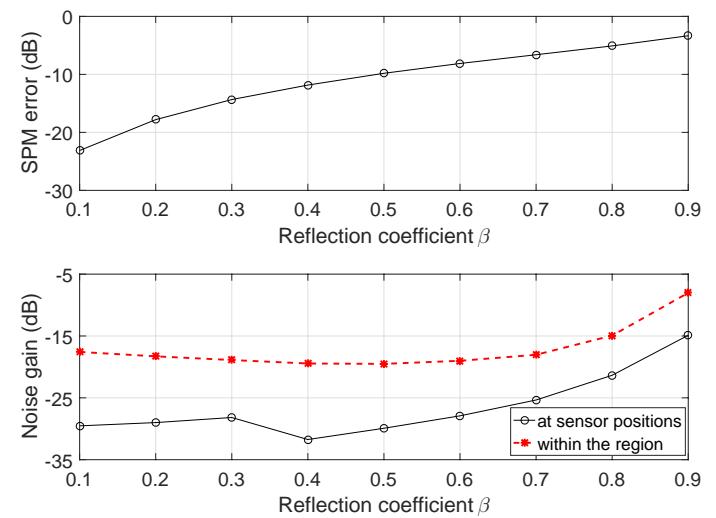

Fig. 11. Performance of WD ANC under different reflection coefficients.

investigated in future work.

In summary, the above results in Figs. 6-10 show a clear advantage of modelling the WD secondary path as a diagonal matrix, that is instead of estimating the whole matrix as in FD and TD ANC only the diagonal entries need to be estimated. This alleviates the well-known non-uniqueness problem in multichannel system identification [35] and allows adaptation 


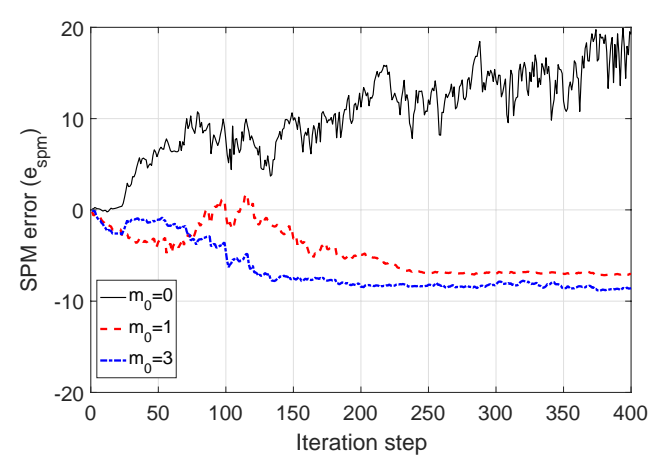

(a)

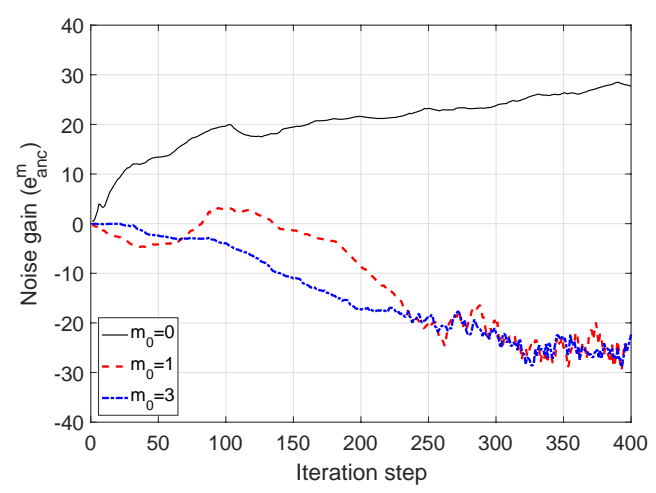

(b)

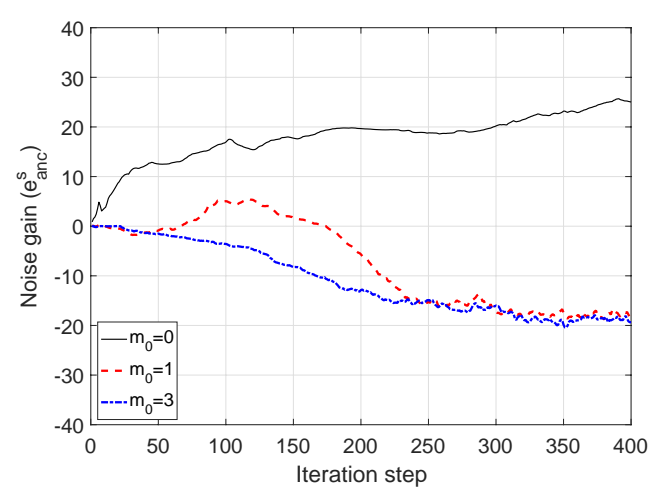

(c)

Fig. 12. Adaptation performance of the proposed WD ANC with the loudspeaker positioning errors $e_{x}=0.3 \mathrm{~m}$. The weighting matrix $\boldsymbol{C}$ is applied with different values of the upper mode limit $m_{0}$. Plots correspond to (a) online SPM error, noise reduction at (b) the microphone positions and (c) within the control region.

with a much lower level of auxiliary noise.

Figure 11 plots the system performance for different reflection coefficients corresponding to the reflection order ranging from 5 to 147 and the reverberation time $T_{60}$ increasing from 110 to $570 \mathrm{~ms}$. As the reflection coefficient $\beta$ increases, the SPM error increases and the noise reduction performance drops as expected. The proposed WD ANC system with the assumption of modelling the WD secondary path as a diagonal matrix however is quite effective for mildly to moderately reverberant environments. For example, even for a high re-

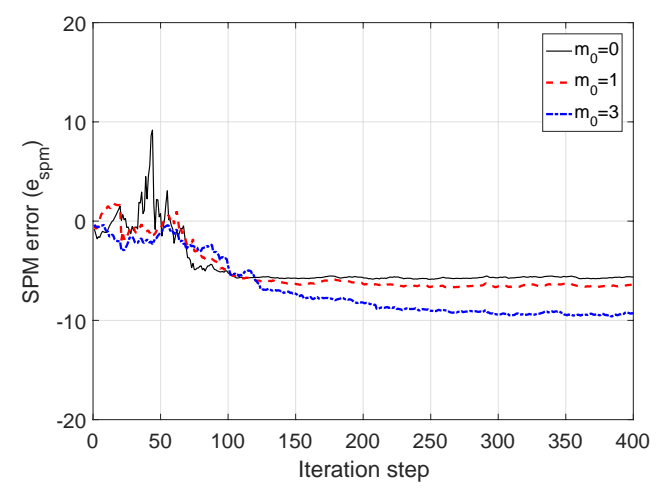

(a)

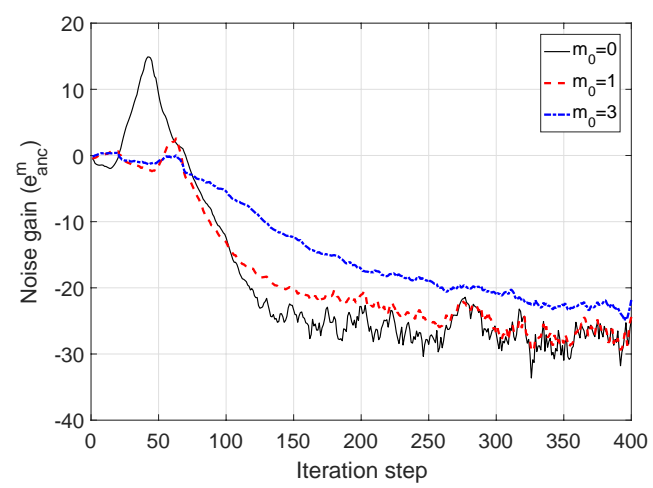

(b)

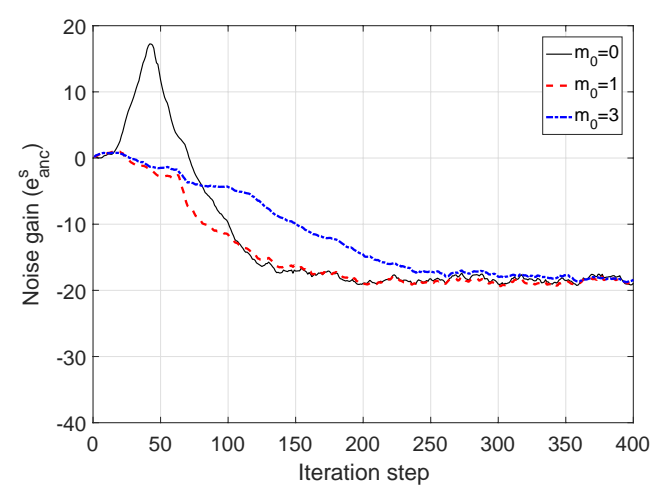

(c)

Fig. 13. Adaptation performance of the proposed WD ANC for nonconcentrically placed loudspeaker and microphone array, i.e., a distance of $\Delta_{x}=0.3 \mathrm{~m}$ between the loudspeaker array and microphone array centre. The weighting matrix $\boldsymbol{C}$ is applied with different values of the upper mode limit $m_{0}$. Plots correspond to (a) online SPM error, noise reduction at (b) the microphone positions and (c) within the control region.

flection coefficient $\beta=0.9$, a high reflection order 147 , and a long reverberation time $T_{60} \approx 570 \mathrm{~ms}$, the SPM error reaches $-3.34 \mathrm{~dB}$, and the noise reduction at microphones and within the region can still be kept at a reasonable level of $-14.88 \mathrm{~dB}$ and $-8.02 \mathrm{~dB}$, respectively.

2) Robustness: In this subsection, we investigate the robustness of the proposed method when dealing with nonideal conditions. As shown in Sec. III-B, a weighting matrix $\boldsymbol{C}$ is applied in online SPM to include off-diagonal components of $\hat{\boldsymbol{H}}_{\mathrm{w}}$ when the loudspeaker array and microphone array are not 


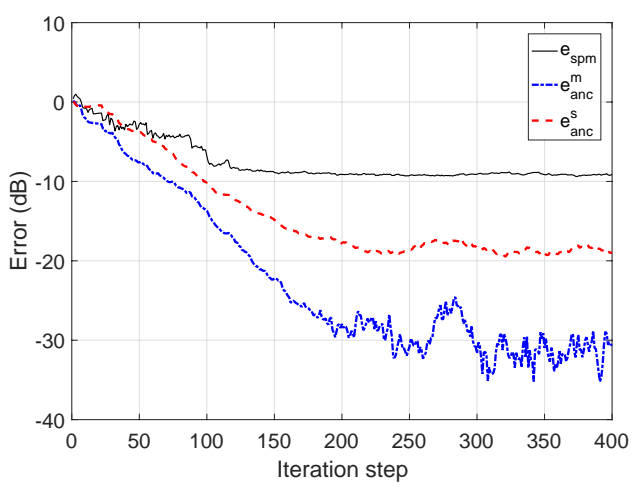

Fig. 14. Adaptation performance of the proposed WD ANC for multi-source noise field. Three primary noise sources, operating at the same frequency 500 $\mathrm{Hz}$, are located at $r_{1}=2 \mathrm{~m} \phi_{1}=45^{\circ}, r_{2}=2.5 \mathrm{~m} \phi_{2}=120^{\circ}$, and $r_{3}=3$ $\mathrm{m} \phi_{3}=270^{\circ}$, respectively. The plot includes online SPM error $e_{\mathrm{spm}}$, noise reduction at the microphone positions $e_{\mathrm{anc}}^{\mathrm{m}}$ and that within the control region $e_{\text {anc }}^{\mathrm{s}}$.

concentrically placed or there are additional positioning errors associated with the loudspeaker and microphone array. In this case, a smaller step size $\mu_{e}=0.1, \mu_{c}=0.1$ is applied.

Plots in Fig. 12 and Fig. 13 show the system performance with different values of the upper mode limit $m_{0}$ applied in the SPM weighting model (12). In Fig. 12, a maximum positioning error $e_{x}=0.3 \mathrm{~m}$ is randomly applied to all loudspeakers. It is clear that the higher the value $m_{0}$ the smaller the SPM error becomes and the better noise reduction results. In Fig. 13, a distance of $\Delta_{x}=0.3 \mathrm{~m}$ between the loudspeaker array and microphone array centre is applied. We can see that modelling the secondary path as a diagonal matrix, i.e., $m_{0}=0$, still achieves the desired results when the same level of auxiliary noise (around $10 \mathrm{~dB}$ lower than the primary noise level) is applied for online SPM. However, using the generalized wave-domain SP model with the upper mode limit $m_{0}=1$ gives more stable results. The differences between these two examples are that in the first case the positioning error $e_{x}$ is not considered as a prior, while in the second case the displacement $\Delta_{x}$ has been incorporated into the wave-domain transform and wave-domain SPM.

Overall, the above examples demonstrate the effectiveness of modelling the wave-domain secondary path as a diagonaldominant matrix when knowing the exact transducer positions and the advantage of the proposed weighting model for systematically incorporating unknown positioning errors into the wave-domain secondary path model.

\section{Multi-source and Multi-Frequency Noise Field}

We next evaluate the proposed wave-domain ANC for cancelling multi-source and multi-frequency noise field. Two cases are investigated, i) multi-source noise field, where three primary noise sources, operating at the same frequency 500 $\mathrm{Hz}$, are located at $r_{1}=2 \mathrm{~m} \phi_{1}=45^{\circ}, r_{2}=2.5 \mathrm{~m} \phi_{2}=120^{\circ}$, and $r_{3}=3 \mathrm{~m} \phi_{3}=270^{\circ}$, respectively; ii) multi-frequency noise field, where a noise source broadcasting $250,375,500 \mathrm{~Hz}$ is located at the point $r_{1}=2 \mathrm{~m} \phi_{1}=45^{\circ}$. We use the system designed for the highest frequency $500 \mathrm{~Hz}$ as in Sec.V-B to

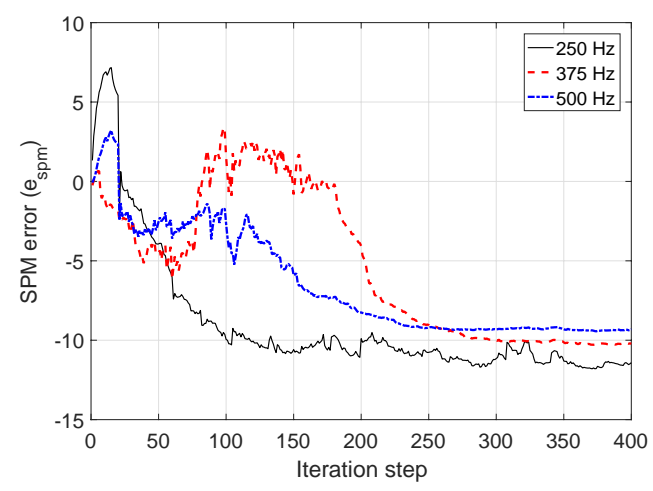

(a)

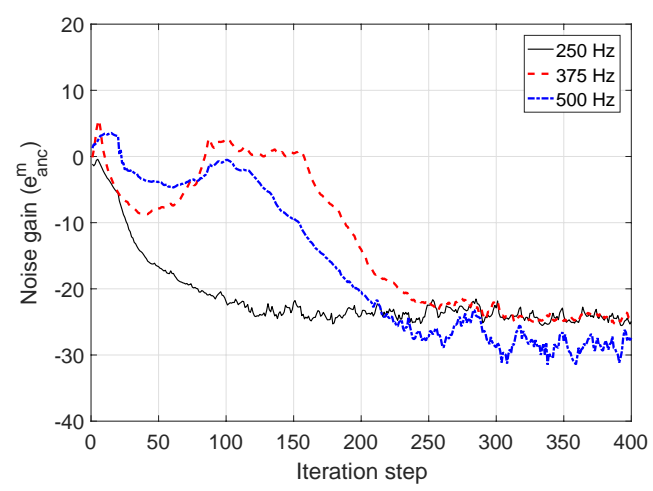

(b)

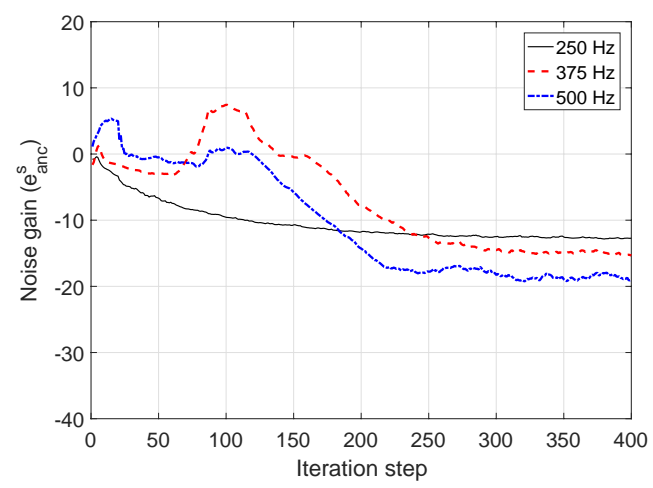

(c)

Fig. 15. Adaptation performance of the proposed WD ANC for multi-frequency noise field. Three noise sources operate at frequency of 250, 375, $500 \mathrm{~Hz}$. Plots correspond to (a) online SPM error, noise reduction at (b) the microphone positions and (c) within the control region.

control this multi-source and multi-frequency noise field. A generalized SPM is adopted with the first minor diagonal term included, i.e., the upper mode limit $m_{0}=1$.

Figures 14 and 15 plot the adaptation performance of controlling each primary noise, including the online SPM, the noise reduction at microphone positions and within the 2D control region. We can see that the system works well for the multi-source case. In the multi-frequency case, especially when all sources are located at the same spatial point, the system still converges but its adaptation performance degrades. This is due to the fact that when dealing with multi-frequency noise, the overall noise level increases as multiple Gold 


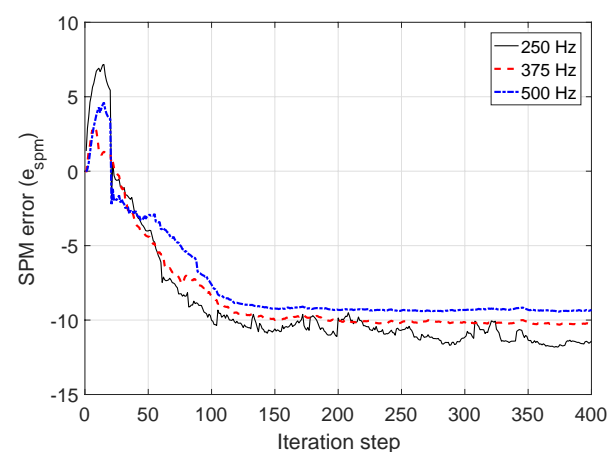

(a)

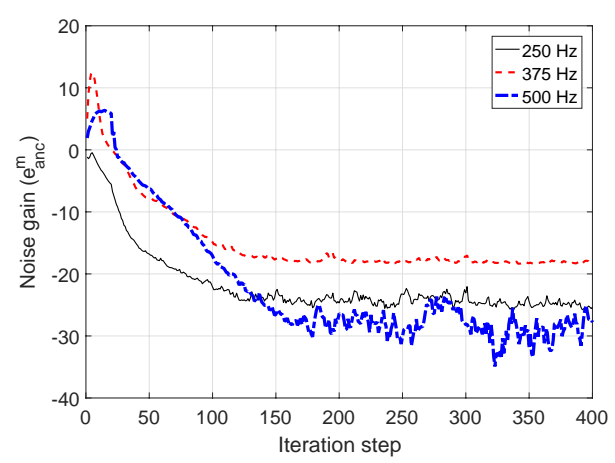

(b)

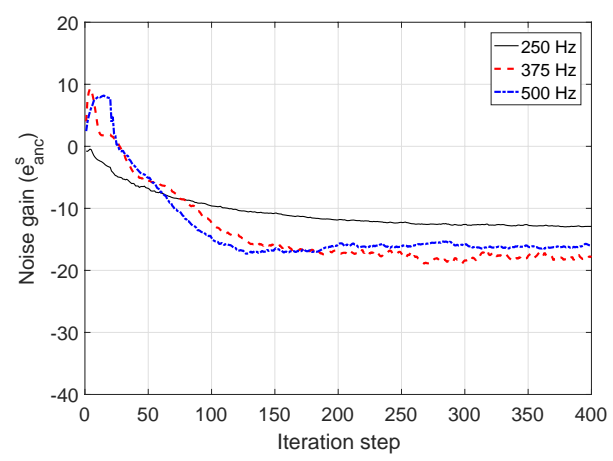

(c)

Fig. 16. Adaptation performance of the proposed WD ANC for multi-source and multi-frequency noise field. Three primary noise sources, operating at the frequency of $250,375,500 \mathrm{~Hz}$, are located at $r_{1}=2 \mathrm{~m} \phi_{1}=45^{\circ}, r_{2}=2.5$ $\mathrm{m} \phi_{2}=120^{\circ}$, and $r_{3}=3 \mathrm{~m} \phi_{3}=270^{\circ}$, respectively. Plots correspond to (a) online SPM error, noise gain at (b) the microphone positions and (c) within the control region.

sequence-modulated auxiliary noises are required for online SPM, and also the spectral leakage caused by the windowing function during the STFT operation increases. The proposed system, which has been verified in the multi-source and multi-frequency case separately, also works efficiently in the combined situation as shown in Fig. 16. The wave-domain representations of the noise field within the control region are the same as in theory, and only the specific decomposition coefficients are different.

\section{CONCLUSION}

Adaptive wave-domain processing for multichannel ANC was presented in this paper to achieve online secondary path modelling and noise cancellation over a spatial region. For a variety of typical loudspeaker and microphone array placement conditions, we showed that the wave-domain secondary path can exploit the property of diagonal dominance. We then proposed a robust wave-domain secondary path model, based on which adaptive algorithms with reduced computational complexity and fast convergence speed were developed. A feedback control strategy was adopted in the proposed system. Simulation results further showed that the proposed wavedomain ANC requires a much lower level of auxiliary noise for online secondary path modelling and achieves significant noise reduction over the entire design region.

\section{REFERENCES}

[1] C. R. Fuller, S. J. Elliott, and P. A. Nelson, Active Control of Vibration, Academic Press, San Diego, CA, 1997.

[2] S. M. Kuo and D. R. Morgan, "Active noise control: A tutorial review," Proceedings of the IEEE, vol. 87, no. 6, pp. 943-973, June 1999.

[3] P. N. Samarasinghe, W. Zhang, and T. D. Abhayapala, "Recent advances in active noise control inside automobile cabins: Toward quieter cars," IEEE Signal Processing Magazine, vol. 33, no. 6, pp. 61-73, Nov. 2016.

[4] H. Sano, T. Inoue, A. Takahashi, K. Terai, and Y. Nakamura, "Active control system for low-frequency road noise combined with an audio system," IEEE Transactions on Speech and Audio Processing, vol. 9, no. 7, pp. 755-763, Oct. 2001.

[5] H. Chen, P. N. Samarasinghe, T. D. Abhayapala, and W. Zhang, "Spatial noise cancellation inside cars: Performance analysis and experimental results," in Proc. IEEE Workshop on Applications of Signal Processing to Audio and Acoustics (WASPAA), New Paltz, NY, Oct. 2015, pp. 1-5.

[6] S. J. Elliott, I. M. Stothers, and P. A. Nelson, "A multiple error LMS algorithm and its application to the active control of sound and vibration," IEEE Transactions on Acoustics, Speech, and Signal Processing, vol. 35, no. 10, pp. 1423-1434, Oct. 1987.

[7] T. Kosaka, S. J. Elliott, and C. C. Boucher, "A novel frequency domain filtered-X LMS algorithm for active noise reduction," in Proc. IEEE International Conference on Acoustics, Speech, and Signal Processing (ICASSP), Munich, Germany, Apr. 1997, pp. 403-406.

[8] S. C. Douglas, "Fast implementations of the filtered-X LMS and LMS algorithms for multichannel active noise control," IEEE Transactions on Speech and Audio Processing, vol. 7, no. 4, pp. 454-465, July 1999.

[9] S. J. Elliott, C. C. Boucher, and P. A. Nelson, "The behavior of a multiple channel active control system," IEEE Transactions on Signal Processing, vol. 40, no. 5, pp. 1041-1052, May 1992.

[10] Y. Kajikawa, W.-S. Gan, and S. M. Kuo, "Recent advances on active noise control: Open issues and innovative applications," APSIPA Transactions on Signal and Information Processing, vol. 1, pp. 1-21, Dec. 2012.

[11] J. Zhang, W. Zhang, and T. D. Abhayapala, "Noise cancellation over spatial regions using adaptive wave domain processing," in Proc. IEEE Workshop on Applications of Signal Processing to Audio and Acoustics (WASPAA), New Paltz, NY, Oct. 2015, pp. 1-5.

[12] C. D. Kestell, B. S. Cazzolato, and C. H. Hansen, "Active noise control in a free field with virtual sensors," Journal of the Acoustical Society of America, vol. 109, no. 1, pp. 232-243, Jan. 2001.

[13] J. M. Munn, B. S. Cazzolato, C. D. Kestell, and C. H. Hansen, "Virtual error sensing for active noise control in a one-dimensional waveguide: Performance prediction versus measurement," Journal of the Acoustical Society of America, vol. 113, no. 1, pp. 35-38, Jan. 2003.

[14] D. J. Moreau, J. Ghan, B. S. Cazzolato, and A. C. Zander, "Active noise control in a pure tone diffuse sound field using virtual sensing," Journal of the Acoustical Society of America, vol. 125, no. 6, pp. 3742-3755, June 2009.

[15] A. Barkefors, S. Berthilsson, and M. Sternad, "Extending the area silenced by active noise control using multiple loudspeakers," in Proc. IEEE International Conference on Acoustics, Speech, and Signal Processing (ICASSP), Kyoto, Japan, May 2012, pp. 325-328.

[16] J. Cheer, Active control of the acoustic environment in an automobile cabin, Ph.D. thesis, University of Southampton, 2012. 
[17] S. Spors and H. Buchner, "An approach to massive multichanne broadband feedforward active noise control using wave-domain adaptive filtering," in Proc. IEEE Workshop on Applications of Signal Processing to Audio and Acoustics (WASPAA), New Paltz, NY, Oct. 2007, pp. 171174.

[18] S. Spors and H. Buchner, "Efficient massive multichannel active noise control using wave-domain adaptive filtering," in Proc. International Symposium on Communications, Control and Signal Processing (ISCCSP), St. Julians, Malta, Mar. 2008, pp. 1480-1485.

[19] P. Peretti, S. Cecchi, L. Palestini, and F. Piazza, "A novel approach to active noise control based on wave domain adaptive filtering," in Proc. IEEE Workshop on Applications of Signal Processing to Audio and Acoustics (WASPAA), New Paltz, NY, Oct. 2007, pp. 307-310.

[20] J. Donley, C. Ritz, and W. B. Kleijn, "Active speech control using wavedomain processing with a linear wall of dipole secondary sources," in Proc. IEEE International Conference on Acoustics, Speech, and Signal Processing (ICASSP), New Orleans, LA, Mar. 2017, pp. 456-460.

[21] J. Zhang, T.D. Abhayapala, W. Zhang, P. N. Samarasinghe, and S. Jiang, "Active noise control over space: A wave domain approach," IEEE/ACM Transactions on Audio, Speech, and Language Processing, vol. 26, no. 4, pp. 774-786, Apr. 2018.

[22] J. Zhang, T. D. Abhayapala, P. N. Samarasinghe, W. Zhang, and S. Jiang, "Sparse complex FxLMS for active noise cancellation over spatial regions," in Proc. IEEE International Conference on Acoustics, Speech, and Signal Processing (ICASSP), Shanghai, China, Mar. 2016, pp. 524 528.

[23] J. Mourjopoulos, "On the variation and invertibility of room impulse response functions," Journal of Sound and Vibration, vol. 102, no. 2, pp. 217-228, Sept. 1985

[24] N. Saito and T. Sone, "Influence of modeling error on noise reduction performance of active noise control systems using filtered-X LMS algorithm," Journal of the Acoustical Society of Japan (E), vol. 17, no. 4, pp. 195-202, 1996.

[25] S. J. Elliot, Signal Processing for Active Control, Academic Press, London, UK, 2000.

[26] S. M. Kuo and D. R. Morgan, Active Noise Control Systems: Algorithms and DSP Implementations, Wiley-Interscience, New York, 1996.

[27] L. J. Eriksson and M. C. Allie, "Use of random noise for on-line transducer modeling in an adaptive active attenuation system," Journal of the Acoustical Society of America, vol. 85, no. 2, pp. 797-802, Feb. 1989.

[28] C. Bao, P. Sas, and H. V. Brussel, "Adaptive active control of noise in 3-D reverberant enclosure," Journal of Sound and Vibration, vol. 161, no. 3, pp. 501-514, Mar. 1993.

[29] S. M. Kuo and D. Vijayan, "A secondary path modeling technique for active noise control systems," IEEE Transactions on Speech and Audio Processing, vol. 5, no. 4, pp. 374-377, July 1997.

[30] M. Zhang, H. Lan, and W. Ser, "Cross-updated active noise control system with online secondary path modeling," IEEE Transactions on Speech and Audio Processing, vol. 9, no. 5, pp. 598-602, July 2001.

[31] M. T. Akhtar, M. Abe, and M. Kawamata, "A new structure for feedforward active noise control system with improved online secondary path modeling," IEEE Transactions on Speech and Audio Processing, vol. 13, no. 5, pp. 1082-1088, Sept. 2005.

[32] A. Carini and S. Malatini, "Optimal variable step-size NLMS algorithms with auxiliary noise power scheduling for feedforward active noise control," IEEE Transactions on Audio, Speech, and Language Processing, vol. 16, no. 8, pp. 1383-1395, Nov. 2008.

[33] P. Davari and H. Hassanpour, "A self-tuning feedforward active noise control system," IEICE Electronics Express, vol. 6, no. 5, pp. 230-236, 2009.

[34] M. T. Akhtar, M. Abe, M. Kawamata, and A. Nishihara, "Online secondary path modeling in multichannel active noise control systems using variable step size," Signal Processing, vol. 88, no. 8, pp. 20192029, Aug. 2008.

[35] J. Benesty, D. R. Morgan, and M. M. Sondhi, "A better understanding and an improved solution to the specific problems of stereophonic acoustic echo cancellation," IEEE Transactions on Speech and Audio Processing, vol. 6, no. 2, pp. 156-165, Mar. 1998.

[36] D. S. Talagala, W. Zhang, and T. D. Abhayapala, "Efficient multichannel adaptive room compensation for spatial soundfield reproduction using a modal decomposition," IEEE/ACM Transactions on Audio, Speech, and Language Processing, vol. 22, no. 10, pp. 1522-1532, Oct. 2014.
[37] M. Schneider and W. Kellermann, "Adaptive listening room equalization using a scalable filtering structure in the wave domain," in Proc. IEEE International Conference on Acoustics, Speech, and Signal Processing (ICASSP), Kyoto, Japan, Mar. 2012, pp. 13-16.

[38] M. Schneider and W. Kellermann, "Multichannel acoustic echo cancellation in the wave domain with increased robustness to nonuniqueness," IEEE/ACM Transactions on Audio, Speech, and Language Processing, vol. 24, no. 3, pp. 518-529, Mar. 2016.

[39] M. Schneider and W. Kellermann, "A direct derivation of transforms for wave-domain adaptive filtering based on circular harmonics," in Proc. European Signal Processing Conference (EUSIPCO), Bucharest, Romania, Aug. 2012, pp. 1034-1038.

[40] W. Zhang, C. Hofmann, M. Bürger, T. D. Abhayapala, and W. Kellermann, "Online secondary path modelling in wave-domain active noise control," in Proc. IEEE International Conference on Acoustics, Speech, and Signal Processing (ICASSP), New Orleans, USA, Mar. 2017, pp. $116-120$.

[41] C. Hofmann and W. Kellermann, "Wave-domain transforms for irregular loudspeaker array topologies," in Proc. DAGA, Nürnberg, Germany, Mar. 2015, pp. 1606-1609.

[42] C. Hofmann and W. Kellermann, "Generalized wave-domain transforms for listening room equalization with azimuthally irregularly spaced loudspeaker arrays," in Proc. IEEE International Conference on Acoustics, Speech, and Signal Processing (ICASSP), Shanghai, China, Mar. 2016, pp. 594-598.

[43] J. Ahrens and S. Spors, "An analytical approach to sound field reproduction using circular and spherical loudspeaker distributions," Acta Acust. Acust., vol. 94, no. 6, pp. 988-999, Nov. 2008.

[44] W. Zhang and T. D. Abhayapala, "2.5D sound field reproduction in higher order Ambisonics," in Proc. International Workshop on Acoustic Signal Enhancement (IWAENC), Juan Ies Pins, French Riviera, Sept. 2014, pp. 342-346.

[45] T. Okamoto, "Analytical approach to $2.5 \mathrm{~d}$ sound field control using a circular double-layer array of fixed-directivity loudspeakers," in Proc. IEEE International Conference on Acoustics, Speech, and Signal Processing (ICASSP), New Orleans, USA, Mar. 2017, pp. 91-95.

[46] F. Winter, J. Ahrens, and S. Spors, "On analytic methods for 2.5-D local sound field synthesis using circular distributions of secondary sources," IEEE/ACM Transactions on Audio, Speech, and Language Processing, , no. 5, pp. 914-926, May 2016.

[47] A. Kuntz, Wave field analysis using virtual circular microphone arrays, Ph.D. thesis, Friedrich-Alexander University Erlangen-Nürnberg (FAU), June 2008.

[48] D. B. Ward and T. D. Abhayapala, "Reproduction of a plane-wave sound field using an array of loudspeakers," IEEE Transactions on Speech and Audio Processing, vol. 9, no. 6, pp. 697-707, Sept. 2001.

[49] E. G. Williams, Fourier Acoustics: Sound Radiation and Nearfield Acoustical Holography, Academic Press, San Diego, CA, 1999.

[50] M. Schneider and W. Kellermann, "A wave-domain model for acoustic MIMO systems with reduced complexity," in Proc. IEEE Workshop on Hands-free Speech Communication and Microphone Arrays (HSCMA), Edinburgh, UK, May 2011, pp. 133-138.

[51] H. Buchner, S. Spors, and W. Kellermann:, "Wave-domain adaptive filtering: acoustic echo cancellation for full-duplex systems based on wave-field synthesis," in Proc. IEEE International Conference on Acoustics, Speech, and Signal Processing (ICASSP), Montreal, Canada, May 2004, vol. 4, pp. 117-120.

[52] S. Spors, H. Buchner, and R. Rabenstein, "A novel approach to active listening room compensation for wave field synthesis using wave-domain adaptive filtering," in Proc. IEEE International Conference on Acoustics, Speech, and Signal Processing (ICASSP), Montreal, Canada, May 2004, vol. 4, pp. 29-32.

[53] D. S. Talagala, W. Zhang, and T. D. Abhayapala, "Active acoustic echo cancellation in spatial soundfield reproduction," in Proc. IEEE International Conference on Acoustics, Speech, and Signal Processing (ICASSP), Vancouver, Canada, May 2013, pp. 620-624.

[54] D. V. Sarwate and M. B. Pursley, "Crosscorrelation properties of pseudorandom and related sequences," Proceedings of the IEEE, vol. 68, no. 5, pp. 593-619, May 1980.

[55] J. Allen and D. Berkley, "Image method for efficiently simulating smallroom acoustics," Journal of the Acoustical Society of America, vol. 65, no. 4, pp. 943-950, Apr. 1979. 